\title{
Formation mechanism of a secondary vortex street in a
}

\section{cylinder wake}

\author{
Hongyi Jiang ${ }^{1 \dagger}$ \\ ${ }^{1}$ School of Engineering, The University of Western Australia, 35 Stirling Highway, \\ Perth, WA 6009, Australia
}

\begin{abstract}
This study identifies and explains the formation mechanisms (FMs) of the secondary vortex street in the far wake of a circular cylinder. Direct numerical simulation and transient growth analysis are performed for Reynolds numbers $(R e)$ in the range of 100 to 200. Unlike most of the earlier studies, which have attributed the formation of the secondary vortices to either the hydrodynamic instability of the mean flow (FM1) or a merging of the two-layered vortices (FM2), the present study demonstrates that both FM1 and FM2 are at play. Specifically, FM1 is observed for $R e=150-160$, whereas FM2 is observed for $R e=200$. Interestingly, both FM1 and FM2 exist (in an alternate manner) for $R e=170$ and 180 . The underlying physical mechanism controlling the manifestation of either FM1 or FM2 is explored. In short, secondary vortices emerging after and before the annihilation of the two-layered vortices are formed from FM1 and FM2, respectively. The variation in the streamwise locations for the emergence of the secondary vortices with $R e$ is quantified and physically explained. Fundamentally, the emergence of the secondary vortices through either FM1 or FM2 is induced by the convective instability of the shear layers in the intermediate wake, which is further induced by an obvious increase in the shear rate of the shear layers as the wake gradually transitions from a primary vortex street to a two-layered vortex street. The general conclusions drawn from a circular cylinder are expected to be applicable to other bluff bodies.
\end{abstract}

\section{Introduction}

A steady incoming flow past a cylindrical bluff body has been a classical topic of research in fluid mechanics owing to its fundamental significance as well as extensive practical applications. An important feature of this type of flow is the generation of vortex shedding and the formation of the classical Kármán vortex street in the near wake of the cylinder. After the discovery of the Kármán vortex street more than a hundred years ago, another experimental study by Taneda (1959) found that the Kármán (primary) vortex street in the near wake of a circular cylinder evolved into a secondary vortex street in the far wake, where the secondary vortices had larger

${ }^{\dagger}$ Correspondence author: hongyi.jiang@uwa.edu.au 
spatial scales than the primary vortices. A more complete picture of the far-wake transition process has been described more recently by e.g. Vorobieff et al. (2002), Kumar and Mittal (2012) and Thompson et al. (2014), who found that the primary vortices in the near wake of the cylinder would first rearrange themselves into a two-layered pattern in the intermediate wake (see also Durgin and Karlsson (1971), Karasudani and Funakoshi (1994) and Dynnikova et al. (2016)), followed by a transition from the two-layered vortex street to the secondary vortex street in the relatively far wake.

The formation mechanism of the secondary vortices in the far wake has already been reported in the literature, although there remains some disagreement. As summarised by Cimbala et al. (1988) and Williamson and Prasad (1993), two different interpretations exist.

(i) Formation mechanism 1 (FM1): the secondary vortices are induced by the hydrodynamic instability of the local mean (time-averaged) flow in the far wake, independent of and not directly resulting from the amalgamation/merging of the primary or two-layered vortices.

(ii) Formation mechanism 2 (FM2): the secondary vortices are formed from the merging of the two-layered vortices.

Specifically, Cimbala et al. (1988), Williamson and Prasad (1993) and Kumar and Mittal (2012) investigated the far wake of a circular cylinder at moderate Reynolds numbers $(R e)$ of approximately 150 , where $\operatorname{Re}(=U D / v)$ is defined based on the free-stream velocity $(U)$, the length scale of the cylinder perpendicular to the free-stream $(D)$ and the kinematic viscosity of the fluid $(v)$, and reported/supported FM1. In addition, Kumar and Mittal (2012) characterised the hydrodynamic instability in FM1 as convective in nature. Cimbala et al. (1988) also suggested that because the secondary vortices were independent of the primary vortices in terms of the scale and frequency, in particular, because the frequency of the secondary vortex street was not half that of the primary vortex street, FM2 seemed questionable.

In contrast, based on experimental observations of the far wake of a circular cylinder at similar Re values, Matsui and Okude (1983) reported FM2 instead. In addition, Matsui and Okude (1983) found that not all of the vortices were paired up (a few were left out) when forming the secondary vortex street, such that even under the condition of FM2, the frequency of the secondary vortex street may not be half that of the primary vortex street. Nevertheless, Cimbala et al. (1988), who supported FM1 over FM2, suspected that the smoke-wire visualisation technique used by Matsui and Okude (1983) may result in misleading vortex merging patterns, because the smoke tracer was introduced upstream of the cylinder and may deviate from the true flow patterns after a long distance of evolution.

An explanation for the identification of both FM1 and FM2 in the literature was proposed by Inoue and Yamazaki (1999), who suggested that the occurrence of FM1 or FM2 is related to frequency forcing of the free-stream flow. Specifically, FM1 occurs when the free-stream flow is unforced (e.g. the cases of Cimbala et al. (1988) and Williamson and Prasad (1993)), while FM2 takes place when the free-stream flow is subjected to frequency forcing (e.g. the case of Matsui and Okude (1983)). 
However, Inoue and Yamazaki's (1999) explanation does not always hold, as FM2 has also been identified for some unforced flows, e.g. flow past a circular cylinder in the extended laminar regime of $R e=200-1000$ (Jiang and Cheng, 2019) and flow past an elliptical cylinder with a cross-sectional aspect ratio $A R$ (the ratio between the streamwise length and transverse length of the body) of 0.25 and $R e=150$ (Thompson et al., 2014).

Vorobieff et al. (2002) investigated the extended laminar regime up to $R e=1000$ through both nearly two-dimensional soap-film experiments and two-dimensional numerical simulations. Although Vorobieff et al. (2002) did not comment on the validity of FM1 or FM2, they suggested that in the extended laminar regime of $R e>$ 200 the formation of the secondary vortices is governed by the wake width of a far-wake similarity solution. However, this mechanism is not discussed further in the present study as the extended laminar regime is beyond the scope of this study.

In light of the earlier studies, the present study aims to address the long-standing argument as to whether FM1 or FM2 is the genuine formation mechanism for the secondary vortex street in the far wake of a circular cylinder under no free-stream forcing. The present study focuses mainly on the $R e$ in the range of $100-200$, which is similar to the ranges of $R e$ investigated by Matsui and Okude (1983), Cimbala et al. (1988), Williamson and Prasad (1993) and Kumar and Mittal (2012). This range of Re is marginally lower than that where the wake transition to three-dimensional states is observed (Williamson, 1996), which justifies the use of two-dimensional direct numerical simulations (DNS) for the present study.

Unlike most of the earlier studies, which took sides of either FM1 or FM2, the present study will show systematically that both FM1 and FM2 are at play for the wake transition to the secondary vortex street (section 3). The underlying physical mechanism controlling the manifestation of either FM1 or FM2 will be explored in section 4. The $R e$-dependence of the streamwise locations for the emergence of the secondary vortices will be explained in section 5 by exploring the fundamental cause for the emergence of the secondary vortices. Finally, major conclusions will be drawn in section 6.

\section{Numerical model}

\subsection{Numerical method}

In the present study, the flow was solved numerically using the spectral/hp element method through an open-source code Nektar++ (Cantwell et al., 2015). The governing equations for the flow are the continuity and incompressible Navier-Stokes equations:

$$
\begin{aligned}
& \nabla \cdot \boldsymbol{u}=0, \\
& \frac{\partial \boldsymbol{u}}{\partial t}+\boldsymbol{u} \cdot \nabla \boldsymbol{u}=-\nabla p+\nu \nabla^{2} \boldsymbol{u},
\end{aligned}
$$

where $\boldsymbol{u}(\boldsymbol{x}, t)=\left(u_{x}, u_{y}\right)(x, y, t)$ is the velocity field, $p(\boldsymbol{x}, t)$ is the kinematic pressure defined as pressure divided by fluid density, $t$ is the time and $v$ is the kinematic 
viscosity. Equations (2.1) and (2.2) were solved by the unsteady Navier-Stokes solver embedded in Nektar++, together with the use of a velocity correction scheme (Karniadakis et al., 1991), a second-order implicit-explicit (IMEX) time-integration scheme and a continuous Galerkin projection. For the rest of the paper, the velocity components $u_{x}$ and $u_{y}$ have been non-dimensionalised by the free-stream velocity $U$.

\subsection{Computational details}

The computational domain and mesh were the same as those used in Jiang and Cheng (2019) for the investigation of the far wake of a circular cylinder in the extended laminar regime of $R e=200-1000$. A rectangular computational domain of $-60 \leq x / D \leq 420$ in the streamwise direction and $-60 \leq y / D \leq 60$ in the transverse direction was used. The centre of the cylinder was placed at $(x, y)=(0,0)$. Fig. 1 shows the macro-element mesh near the cylinder. Specifically, the cylinder perimeter was discretized equally into 48 macro-elements, and the radial size of the first layer of macro-elements next to the cylinder surface was $0.0055 D$. A relatively high mesh resolution was adopted in the wake region, where the streamwise size of the macro-elements at the wake centreline $(y=0)$ increased gradually from $0.1875 D$ at $x / D=2$ to $2.5 \times 0.1875 D$ at $x / D=400$. A total of 46,092 macro-elements were used in the domain. Each macro-element was further subdivided using 5th-order Lagrange polynomials (denoted $N_{p}=5$ ) for the quadrilateral expansions.

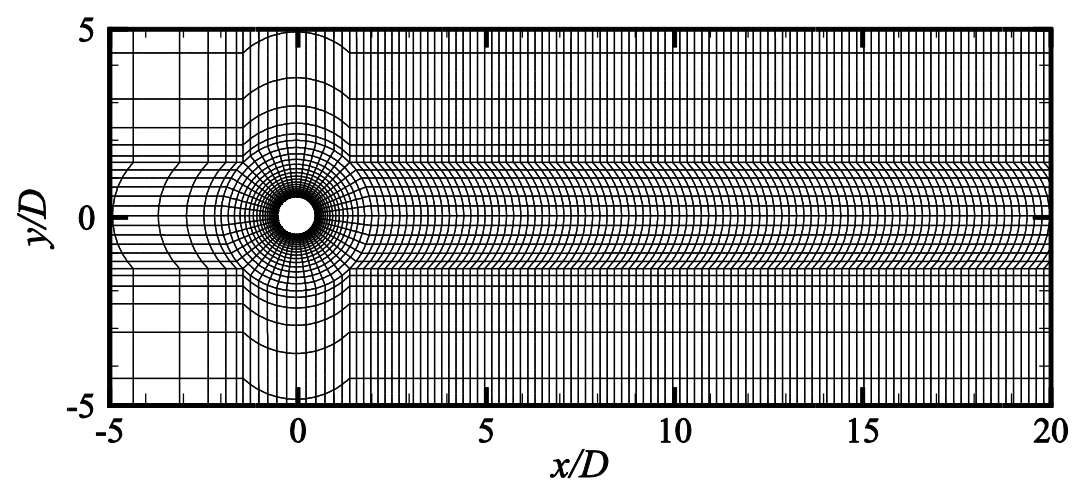

Fig. 1. Macro-element mesh near the cylinder.

The boundary conditions were specified as a uniform velocity $\left(u_{x}, u_{y}\right)=(U, 0)$ at the inlet and transverse sides, a zero normal velocity gradient at the outlet, and a no-slip condition at the cylinder surface. The pressure was specified as a reference value of zero at the outlet, and a high-order Neumann condition (Karniadakis et al., 1991) at all other boundaries. The internal flow followed an impulsive start. The time-step size was chosen based on a Courant-Friedrichs-Lewy (CFL) limit of 0.5.

Because the flow in the far wake of the cylinder is irregular over time (e.g. Kumar and Mittal, 2012), in the present study, each case was simulated for at least 3000 non-dimensional time units (defined as $t^{*}=t U / D$ ). The first 1800 time units ensured that the entire wake had become fully developed. This was followed by at least another 1200 time units (at least 200 primary vortex shedding periods) for the 
purpose of statistics and analysis of the fully developed flow.

The present DNS were conducted on a Cray XC40 system supercomputer. For each case with a total of 46,092 macro-elements coupled with $N_{p}=5,144$ processors were used for parallel computation. The wall-clock time for each numerical simulation up to 3000 non-dimensional time units was approximately 165 hours.

\subsection{Mesh convergence and validation}

A mesh dependence study was conducted at $R e=200$, the largest $R e$ simulated in the present study. Three variations to the reference mesh introduced in section 2.2 were examined, and some key results obtained with the four cases are listed in Table 1. The Strouhal number $(S t)$ and the drag and lift coefficients $\left(C_{D}\right.$ and $C_{L}$, respectively) are defined as

$$
\begin{aligned}
& S t=\frac{f_{L} D}{U}, \\
& C_{D}=\frac{F_{D}}{\frac{1}{2} \rho U^{2} D}, \\
& C_{L}=\frac{F_{L}}{\frac{1}{2} \rho U^{2} D},
\end{aligned}
$$

where $f_{L}$ is the frequency of the fluctuating lift force, while $F_{D}$ and $F_{L}$ are the drag and lift forces per unit span length, respectively. The time-averaged drag coefficient $\left(\overline{C_{D}}\right)$

and root-mean-square lift coefficient $\left(C_{L}^{\prime}\right)$ are reported in Table 1 . Table 1 also summarises the streamwise location for the wake evolution from the primary to the two-layered vortex street $\left(x_{t r 1}\right)$ and the range of streamwise locations for the occurrence of vortex merging $\left(x_{t r 2}\right)$. Specifically, $x_{t r 1}$ was quantified as the streamwise location where the time-averaged $u_{y}$ field displayed a local maximum and a local minimum at the two sides of the wake centreline (Jiang and Cheng, 2019), while the range of $x_{t r 2}$ was determined through visualisation of more than 60 instantaneous vorticity fields over a range of more than 2000 non-dimensional time units.

As shown in Table 1, cases 1 to 3 focussed on the adequacy of the mesh resolution by varying the $N_{p}$ value. With the increase in $N_{p}$, the time-step size $(\Delta t)$ was reduced accordingly (see Table 1 ) to satisfy the CFL limit of 0.5. Cases 1 to 3 showed that the numerical results generally converged at $N_{p} \geq 4$, which suggested that the use of $N_{p}=5$ in the present study was adequate. Case 4 examined the adequacy of the computational domain size through increasing the lengths from the cylinder centre to the upstream and transverse boundaries from $60 \mathrm{D}$ used in case 1 to $90 \mathrm{D}$. The close agreement in the numerical results between cases 4 and 1 suggested that the computational domain size used in case 1 was adequate. Table 1 also shows that the $S t$, $\overline{C_{D}}$ and $C_{L}{ }^{\prime}$ values calculated for case 1 agreed well with those reported by 
Posdziech and Grundmann (2001), with the relative differences being less than $0.2 \%$.

Table 1. Mesh dependence check of key results for $R e=200$.

\begin{tabular}{lllllll}
\hline Case description & \multirow{2}{*}{$\Delta t$} & $S t$ & $\overline{C_{D}}$ & $C_{L}{ }^{\prime}$ & $x_{t r 1} / D$ & $x_{t r 2} / D$ \\
\hline 1. $N_{p}=5$ (reference case) & 0.0024 & 0.1956 & 1.3271 & 0.4763 & 28.7 & $77-110$ \\
2. $N_{p}=4$ & 0.0038 & 0.1956 & 1.3271 & 0.4765 & 28.7 & $77-110$ \\
3. $N_{p}=3$ & 0.0066 & 0.1956 & 1.3273 & 0.4762 & 28.7 & $86-120$ \\
4. Increase in the domain size & 0.0024 & 0.1954 & 1.3232 & 0.4754 & 28.9 & $79-110$ \\
Posdziech and Grundmann (2001) & & 0.1954 & 1.3251 & 0.4757 & & \\
\hline
\end{tabular}

In addition, a commonly reported validation of the far-wake pattern of a circular cylinder is the time-averaged streamwise velocity profile sampled along the wake centreline at $R e=150$ (Cimbala et al., 1988; Williamson and Prasad, 1993; Kumar and Mittal, 2012; Thompson et al., 2014), which is shown in Fig. 2. Fig. 2 also shows the present DNS results with $N_{p}=3$ and 5, which were nearly identical. In addition, the present DNS results agreed well with the recent numerical results of Kumar and Mittal (2012) and Thompson et al. (2014).

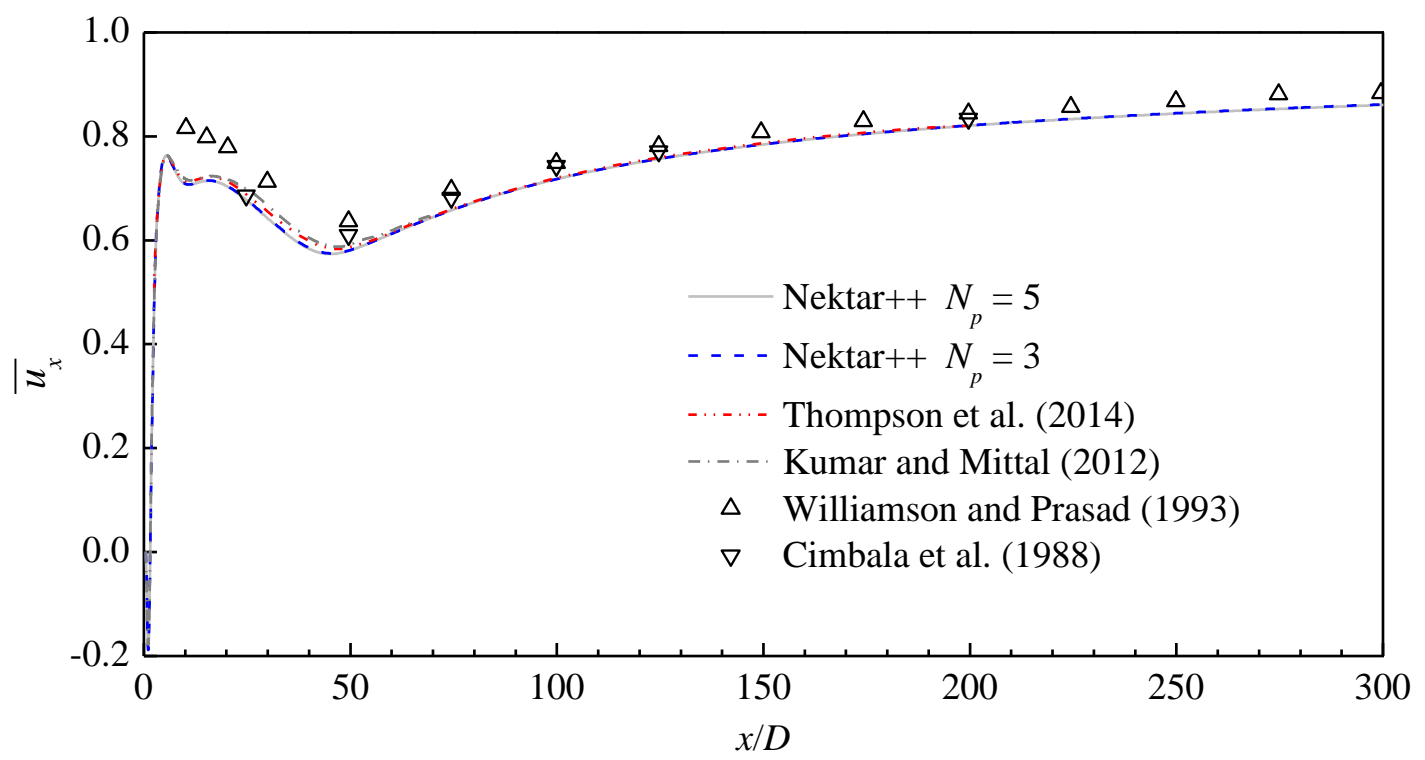

Fig. 2. Time-averaged streamwise velocity profile sampled along the wake centreline for $R e=150$.

Furthermore, Fig. 3 shows the streamwise variation of the amplitude of $u_{y}$ at the primary vortex shedding frequency $\left(f_{K}\right)$ for $R e=150$, which was determined through the fast Fourier transform (FFT) of the time history of $u_{y}$ at various streamwise locations along the wake centreline. A curve fitting of the measured results over $20 \leq$ $x / D \leq 160$ indicated an exponential decay of the primary vortices at a rate of 0.0241 decades $/ D$, which agreed well with the rates of 0.0246 and 0.0235 decades $/ D$ reported by Cimbala et al. (1988) and Kumar and Mittal (2012), respectively. 


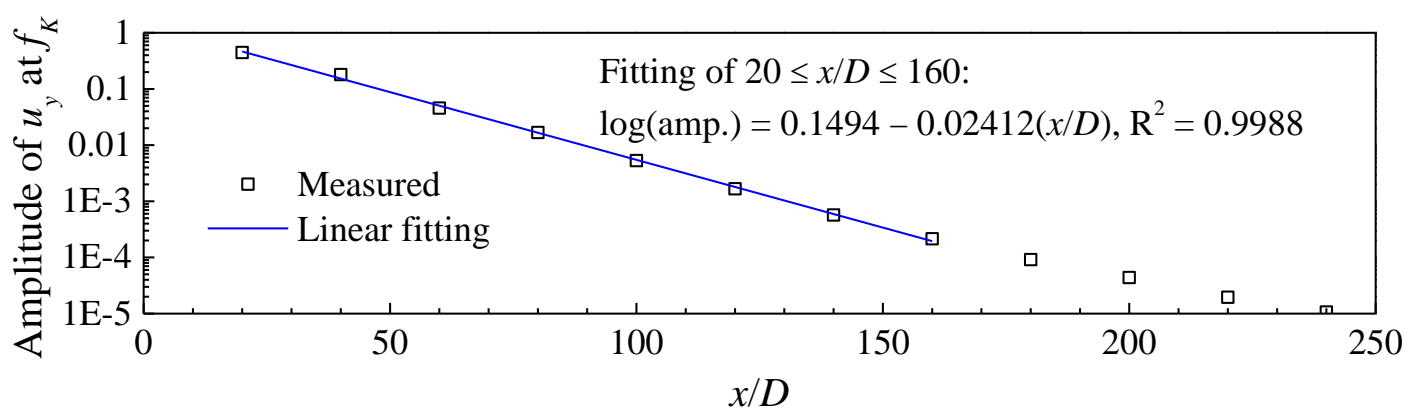

Fig. 3. Streamwise variation of the amplitude of $u_{y}$ at $f_{K}$ for $R e=150$. In the curve fitting, $\mathrm{R}^{2}$ is the coefficient of determination.

\subsection{Transient growth analysis}

In addition to the DNS, transient growth analysis was adopted in section 5 to quantify the optimal growth of the perturbation energy in the base flow. The transient growth analysis generally followed the procedures introduced by Blackburn et al. (2008) and Thompson (2012). Specifically, the transient growth analysis investigates a time-invariant base flow $(\overline{\boldsymbol{u}}(x, y), \bar{p}(x, y))$ seeded with an infinitesimal perturbation field $\left(\boldsymbol{u}^{\prime}(x, y, t), p^{\prime}(x, y, t)\right)$, i.e.

$\boldsymbol{u}=\overline{\boldsymbol{u}}+\boldsymbol{u}^{\prime}, \quad p=\bar{p}+p^{\prime}$

Both the full flow $(\boldsymbol{u}, p)$ and the base flow satisfy the Navier-Stokes equations, which gives rise to the linearised Navier-Stokes equations for the perturbation field:

$\nabla \cdot \boldsymbol{u}^{\prime}=0$

$\frac{\partial \boldsymbol{u}^{\prime}}{\partial t}+\overline{\boldsymbol{u}} \cdot \nabla \boldsymbol{u}^{\prime}+\boldsymbol{u}^{\prime} \cdot \nabla \overline{\boldsymbol{u}}=-\nabla p^{\prime}+v \nabla^{2} \boldsymbol{u}^{\prime}$

According to Barkley et al. (2008), Blackburn et al. (2008) and Thompson (2012), the boundary conditions for the perturbation field are $\boldsymbol{u}^{\prime}=\mathbf{0}$ for all boundaries.

The aim of the transient growth analysis was to determine an optimal initial perturbation field that would give rise to the optimal/maximum energy growth after time evolution of the perturbation over a fixed non-dimensional time interval $\tau$, i.e. from introducing the initial perturbation at $t^{*}=0$ to evaluating the energy growth at $t^{*}$ $=\tau$. For an arbitrary initial perturbation, the energy growth over $\tau$ is calculated as $E\left(t^{*}\right.$ $=\tau) / E\left(t^{*}=0\right)$, where

$E=\frac{1}{2} \int_{\Omega}\left(u_{x}^{\prime 2}+u_{y}^{\prime 2}\right) \mathrm{d} \Omega$,

and $\boldsymbol{u}^{\prime}=\left(u_{x}{ }^{\prime}, u_{y}{ }^{\prime}\right)$ is the perturbation velocity, and $\Omega$ is the area of the computational domain. Among all possible initial perturbations, there exists an optimal initial perturbation that would lead to the optimal/maximum energy growth $G(\tau)$ over $\tau$, 
where $G(\tau)$ can be expressed as

$G(\tau)=\max \frac{E\left(t^{*}=\tau\right)}{E\left(t^{*}=0\right)}$,

In practice, $G(\tau)$ can be determined by calculating the leading eigenvalue of the operator $A^{*}(\tau) A(\tau)$, where $A(\tau)$ and $A^{*}(\tau)$ are the evolution operator and adjoint evolution operator, respectively, and are obtained by integrating the linear system forward from $t^{*}=0$ to $t^{*}=\tau$ and then integrating the adjoint linear system backward from $t^{*}=\tau$ to $t^{*}=0$ (Blackburn et al., 2008). The eigenvalue problem was solved numerically through the transient growth framework embedded in Nektar++, where the eigenvalues were calculated through the modified Arnoldi algorithm (Tuckerman and Barkley 2000; Barkley et al. 2008; Blackburn et al., 2008). In addition, the same velocity correction scheme, time-integration scheme and Galerkin projection as those used for the nonlinear DNS were adopted here for the linear system.

The transient growth analysis used a macro-element mesh that was coarser than that used for the DNS (and the base flow), so as to reduce the computational cost, with the requisite that the accuracy was unaffected. Specifically, the size of each macro-element was increased by four times in both the $x$ - and $y$-directions, while the general topology remained unchanged. A mesh convergence check was performed at $\operatorname{Re}=150$, where $G(\tau)$ was calculated for two cases $\tau=1$ and 100. Because the $G(\tau)$ values calculated with the default and coarser meshes showed relative differences of less than $1 \%$, the coarser mesh was used for the transient growth analysis in section 5 . The base flow, which was calculated with the default mesh, was mapped to the coarser mesh for the transient growth analysis.

By using the time-averaged base flow of the entire computational domain, the present transient growth analysis found that the most unstable eigenmode developed in the immediate neighbourhood of the cylinder, which was similar to the results observed by Kumar and Mittal (2012). To investigate the transient growth in the intermediate wake, the computational domain for the transient growth analysis was truncated to $x / D \geq 10$ (and the boundary condition for the truncated boundary was $\boldsymbol{u}^{\prime}=$ 0). Kumar and Mittal (2012) showed that for $R e=150$, the transient growth in the intermediate wake is not affected by the truncation of the domain up to $x / D \geq 30$ (while influence appears for truncations of $x / D \geq 40$ and more). The limit of $x / D=30$ appears close to the transition to the two-layered vortex street at $x / D \sim 28$, which suggests that the two-layered wake was most responsible for the transient growth.

\section{Two formation mechanisms for the secondary vortex street}

\subsection{Formation mechanism 2 (FM2) for $R e=200$}

The time evolution of the vortices at $R e=200$ is illustrated by a typical sequence of instantaneous vorticity fields captured in the range of $t^{*}=\left(2500+12 T_{1}^{*}\right)-(2500$ $\left.+18 T_{1}^{*}\right)$ in Fig. 4, where $T_{1}^{*}\left(=T_{1} U / D\right)$ is the non-dimensional primary vortex shedding period. The vorticity $\omega$ is defined in a non-dimensional form: 
$\omega=\left(\frac{\partial u_{y}}{\partial x}-\frac{\partial u_{x}}{\partial y}\right) \frac{D}{U}$

(a)

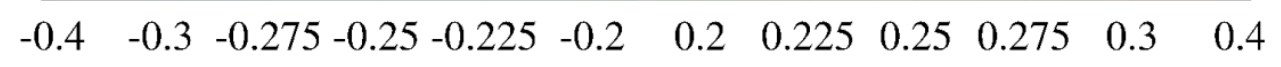

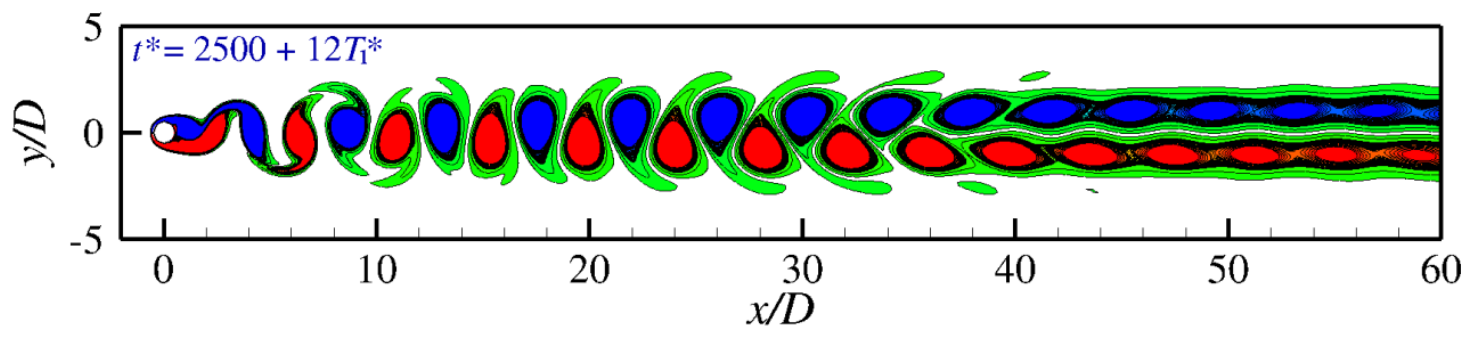

(b)

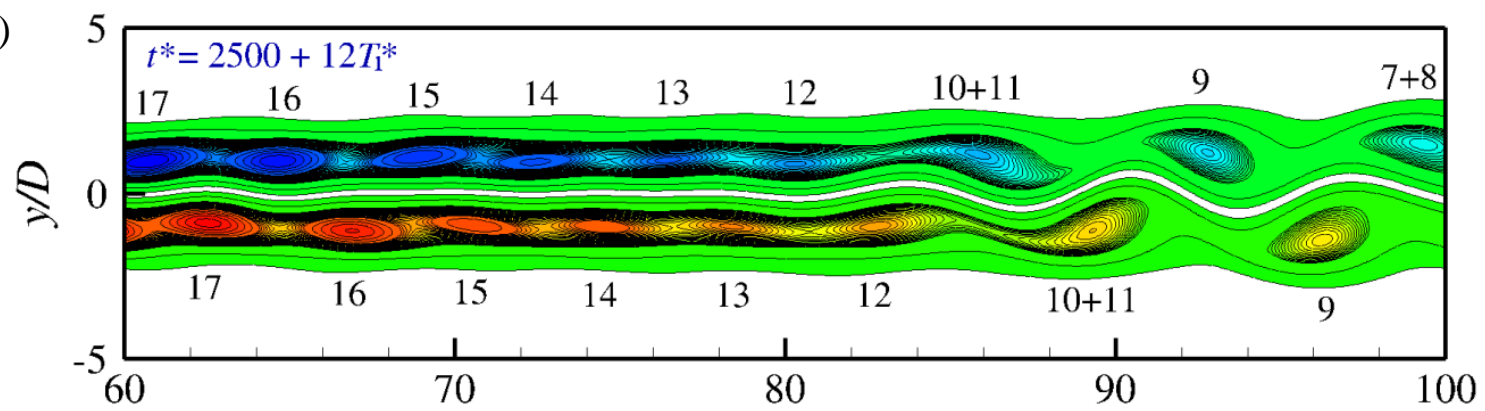

(c)

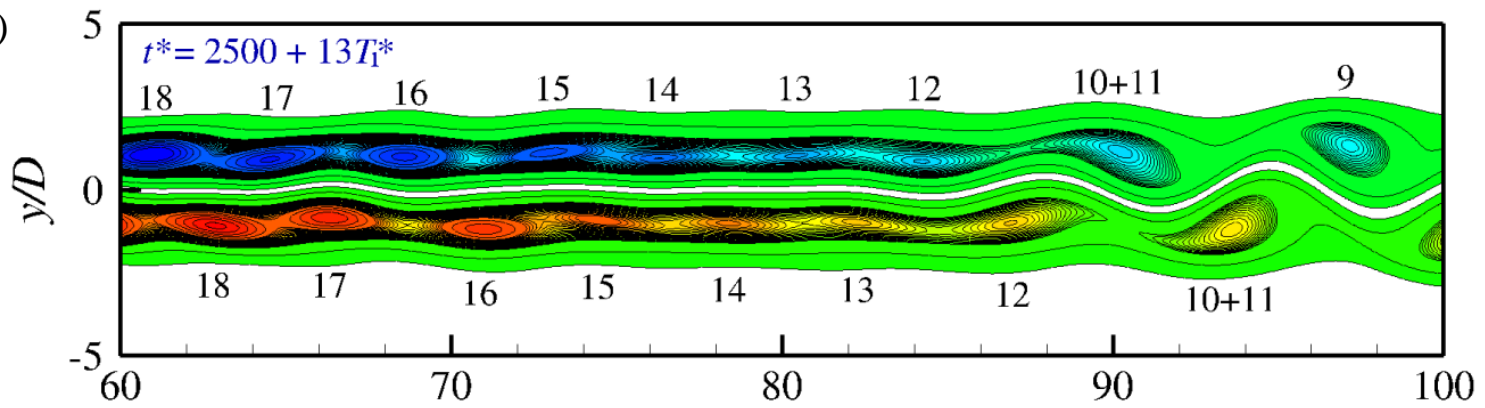

(d)

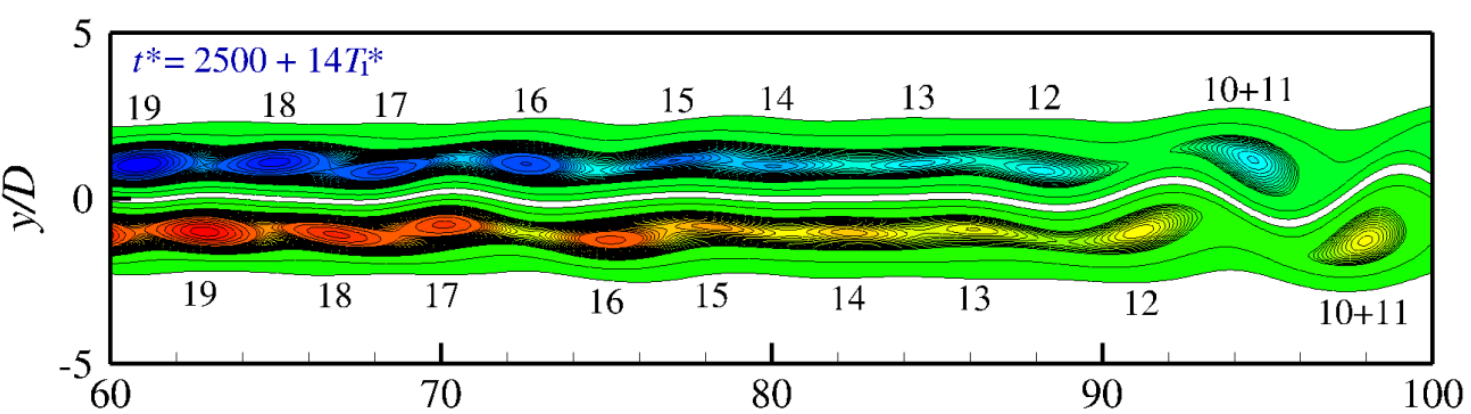


(e)

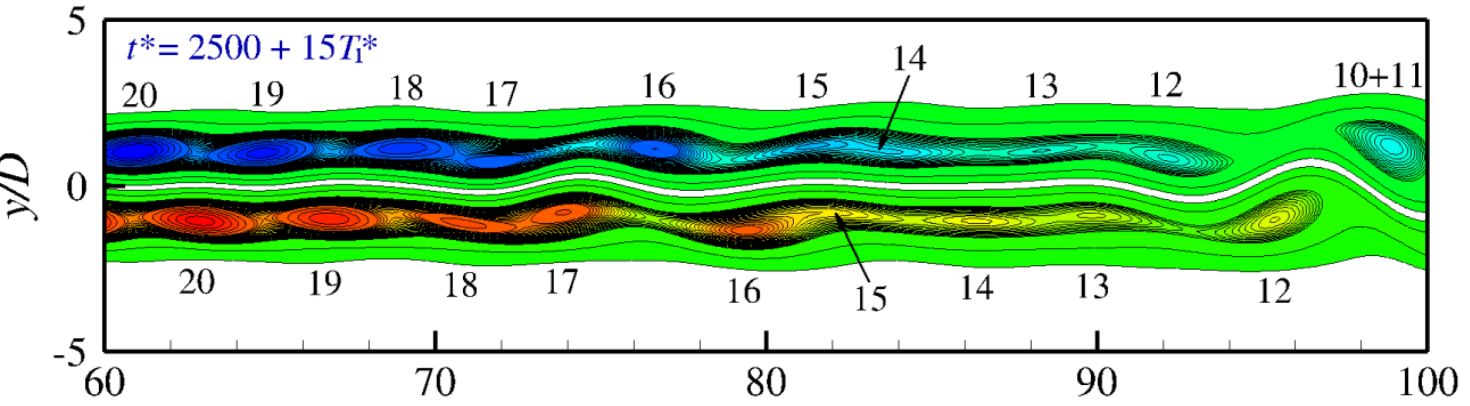

(f)

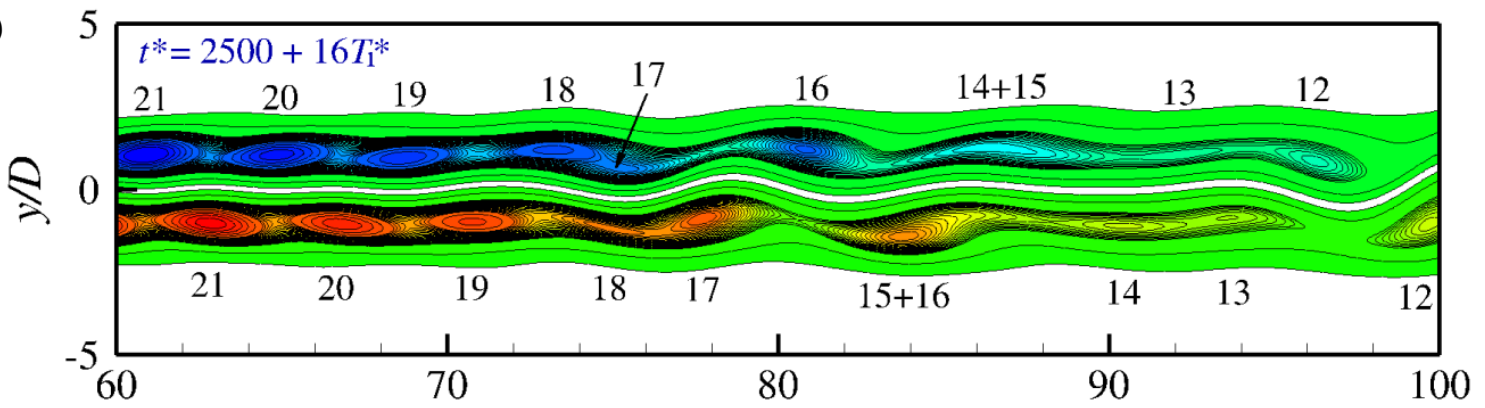

$(\mathrm{g})$

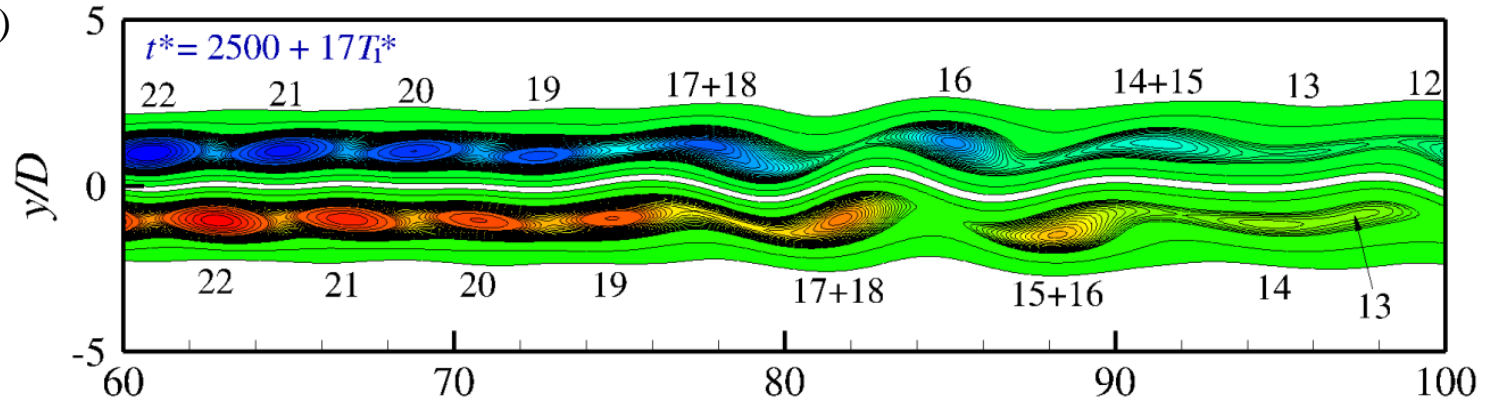

(h)

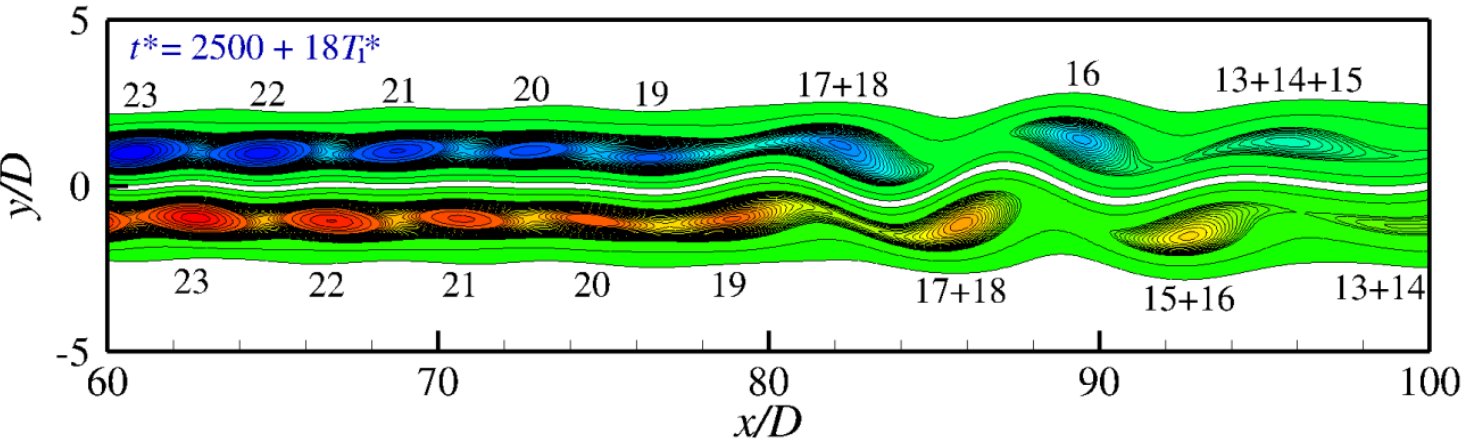

Fig. 4. A short sequence of instantaneous vorticity fields at $R e=200$. The vorticity contours are shown from -0.4 to 0.4 . The vorticity ranges of -0.3 to -0.2 as well as 0.2 to 0.3 are shown with a small interval of 0.005 to highlight the vortex merging process. The same vortices that appear in different snapshots are labelled with the same numbers.

According to flow visualisation, the vorticity fields are largely periodic for $x / D \lesssim$ 60. For example, the first negative vortex observed in Fig. 4(b-h) at $x / D \sim 60-62$ was highly repeatable over the vortex shedding periods shown in Fig. 4(b-h). Therefore, the highly repeatable vorticity field for $x / D \leq 60$ is illustrated in Fig. 4(a) at a single time instant only. The main feature shown in Fig. 4(a) was the breakdown of 
the primary vortex street into the two-layered pattern at $x / D \sim 28.7$ (Table 1).

The two-layered vortices became irregular at $x / D \gtrsim 60$ (Fig. $4 \mathrm{~b}-\mathrm{h}$ ). To facilitate tracing the time evolution of the vortices in Fig. $4(\mathrm{~b}-\mathrm{h})$, the same vortices that appeared in different snapshots are labelled with the same numbers. The labels above the layer of negative vortices correspond to the negative vortices, while the labels below the layer of positive vortices correspond to the positive vortices. Fig. 4(b-h) focuses on the time evolution of a short sequence of vortices, namely the positive and negative vortices labelled from 12 to 18 . At the first time instant $t^{*}=2500+12 T_{1} *$ (Fig. 4b), the positive/negative vortices 10 and 11 had already merged into a positive/negative vortex called 10+11, while vortices labelled 12 and above remained intact.

Fig. 4(b-h) shows clear evidence of the vortex merging over a range of six vortex shedding periods. Some interesting observations are summarised as follows.

(i) Negative vortices 14 and 15 gradually show the tendency of merging in Fig. 4(b-e) and finally merge into a negative vortex $14+15$ in Fig. 4(f). The newly-merged negative vortex 14+15 further absorbs negative vortex 13 in Fig. $4(\mathrm{f}-\mathrm{h})$, which results in a further merged negative vortex $13+14+15$ in Fig. 4(h). The merging of a total of three vortices is a new phenomenon that has not been observed in previous studies. For example, at $R e=300$ and 600 the merging of two same-sign vortices would result in the immediate development of the secondary vortex street (Jiang and Cheng, 2019). The merging of three vortices at $R e=200$ suggests that at relatively small $R e$ values some merged vortices may still be relatively weak and remain in the two parallel shear layers, and a second merging event is required for this vortex to become a secondary vortex.

(ii) Fig. 4(h) shows that there are three merged positive vortices $13+14,15+16$ and $17+18$. An interesting finding is that vortex merging may not always follow the sequence of vortices in the two-layered vortex street. Here positive vortices 15 and 16 merge first (Fig. 4e,f), followed by positive vortices 17 and 18 (Fig. 4f,g) and 13 and 14 (Fig. 4g,h). This phenomenon arises from the interaction between the two parallel rows of vortices. The tendency of merging of the positive vortex 15 into 16 in Fig. 4(c-e) is induced by the tendency of the merging of negative vortices 14 and 15 just above it. Subsequent merging of positive vortices 17 and 18 and negative vortices 17 and 18 in Fig. 4(f,g), as well as the merging of positive vortices 13 and 14 and negative vortices 13 and $14+15$ in Fig. 4(g,h), also arise from the interaction between the two parallel rows of vortices at similar streamwise locations.

(iii) Not all vortices are involved in the merging process. For example, negative vortices 12 and 16 and positive vortex 12 are left out as single vortices in the secondary vortex street. This finding is consistent with that reported by Matsui and Okude (1983).

In addition to the short sequence of $6 T_{1}^{*}$ illustrated in Fig. 4 , the time evolution of the vortices was examined for an extended period of $70 T_{1}^{*}$, i.e. $t^{*}=\left(2500+1 T_{1} *\right)$ $-\left(2500+71 T_{1}^{*}\right)$. The numbers of vortices observed in the primary and secondary 
vortex streets are summarised in Table 2. As shown in Table 2, for each type of vortex in the secondary vortex street (i.e. single vortex, merging of two vortices and merging of three vortices), the numbers of positive and negative vortices were not the same. This fact, together with the observations (i) and (ii) summarised previously, suggested that at small $R e$ values (of $R e \lesssim 200$ ), the relatively weak two-layered vortices result in more freedom for the vortex merging process. Therefore, the vortex merging process is increasingly irregular with decreasing $R e$. Nevertheless, the total number of times of vortex merging, calculated as $19+7 \times 2$ for the positive vortices and $17+8 \times 2$ for the negative vortices, was both 33 over the period of $70 T_{1}{ }^{*}$. The number of times of merging for the positive and negative vortices was the same because vortex merging is a result of the interaction between the two parallel rows of vortices at similar streamwise locations. It was also confirmed through visualisation of the time evolution of the vortices that each merging of the positive vortices was indeed accompanied by a merging of the negative vortices nearby, which was similar to the $3 \times 2$ times of vortex merging illustrated in Fig. 4 and discussed in observation (ii) above.

Table 2. Numbers of vortices observed in the primary and secondary vortex streets for $R e=200$ over 70 primary vortex shedding periods.

\begin{tabular}{|c|c|c|c|c|c|}
\hline & \multirow{2}{*}{$\begin{array}{l}\text { Number of vortices } \\
\text { in the primary } \\
\text { vortex street }\end{array}$} & \multicolumn{4}{|c|}{ Number of vortices in the secondary vortex street } \\
\hline & & $\begin{array}{l}\text { Single } \\
\text { vortex }\end{array}$ & $\begin{array}{l}\text { Merging of } \\
\text { two vortices }\end{array}$ & $\begin{array}{l}\text { Merging of } \\
\text { three vortices }\end{array}$ & $\begin{array}{l}\text { Total } \\
\text { vortices }\end{array}$ \\
\hline Positive vortices & 70 & 11 & 19 & 7 & 37 \\
\hline Negative vortices & 70 & 12 & 17 & 8 & 37 \\
\hline
\end{tabular}

The same number of merging times of 33 results in the same number of positive and negative vortices $70-33=37$ in the secondary vortex street. The 37 positive and 37 negative vortices in the secondary vortex street, as summarised in Table 2, are further visualised in Fig. 5. The label on each of the secondary vortex indicates its origin in the primary and two-layered vortex streets (i.e. before the merging). The labels above and below the vortices correspond to the negative and positive vortices, respectively. The same vortices that appeared in different snapshots are labelled with the same numbers. The labels used in Fig. 5 and Fig. 4 are consistent. For example, the negative secondary vortex $13+14+15$ shown in Fig. 5(a,b) and Fig. 4(h) was the same vortex, and was merged from negative vortices 13, 14 and 15 in Fig. 4(b-d). Fig. 5 shows clearly the type of each secondary vortex (i.e. single vortex, merging of two vortices and merging of three vortices), as previously summarised in Table 2. 
(a)

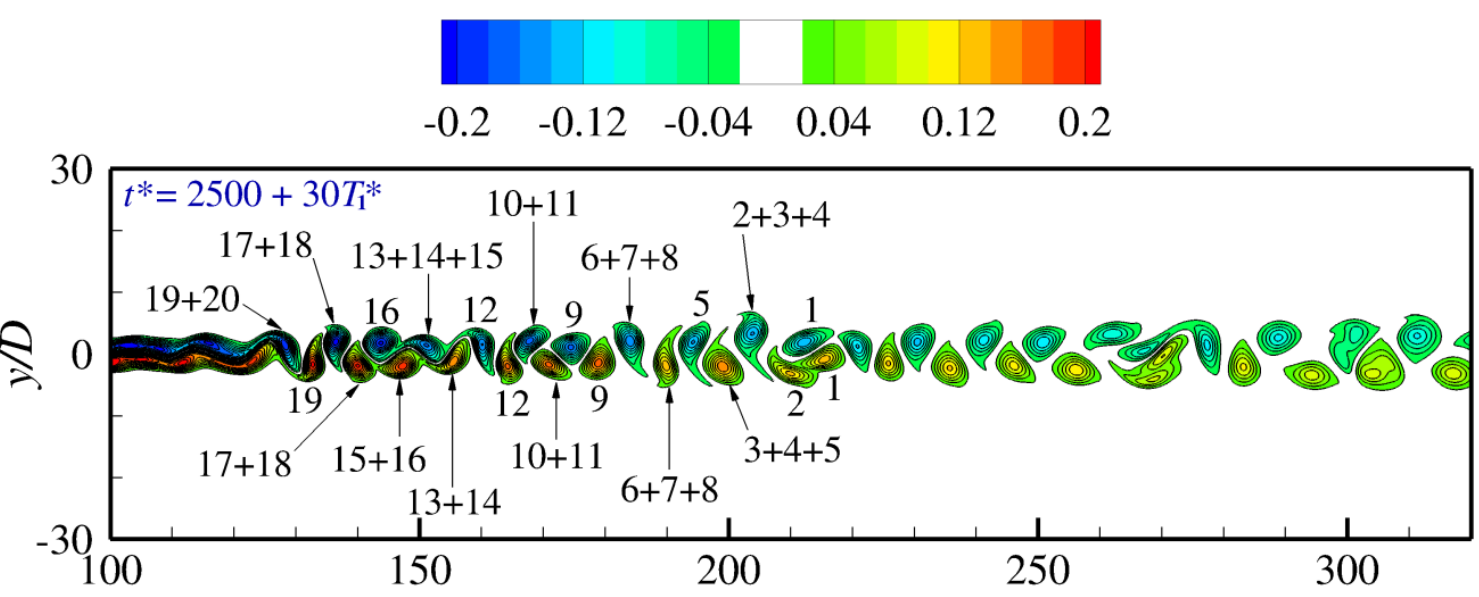

(b)

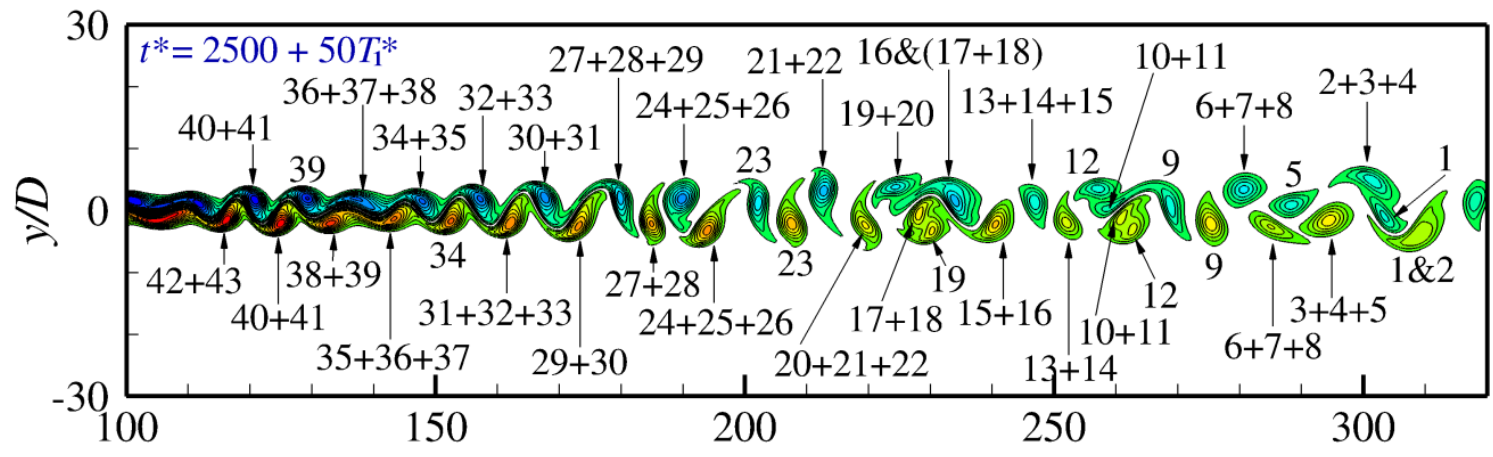

(c)

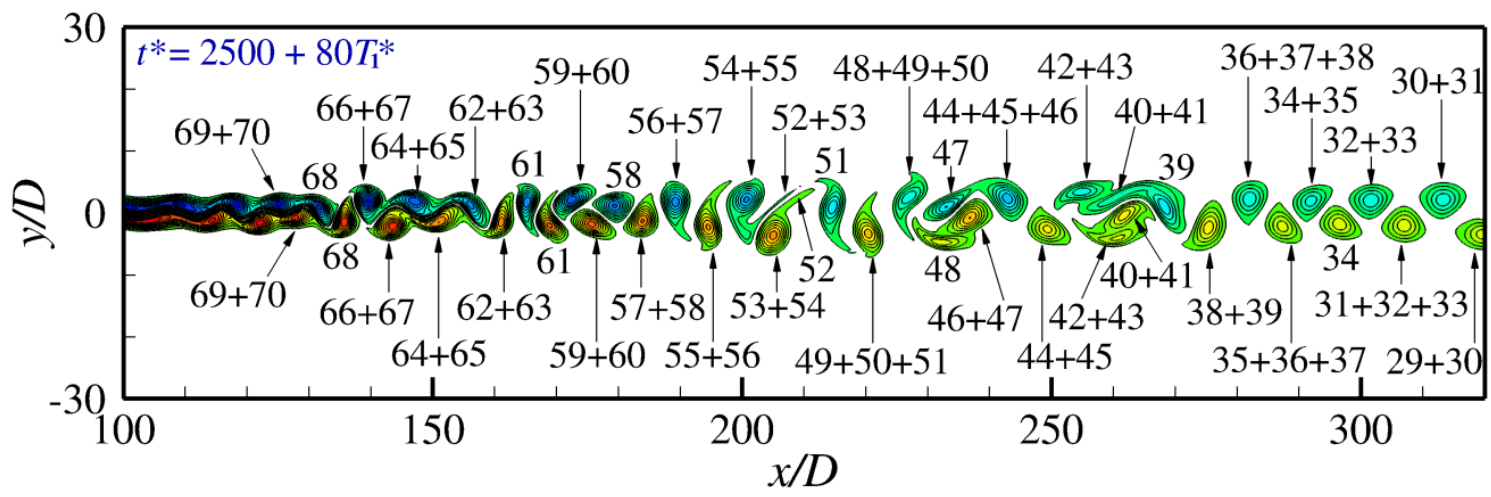

Fig. 5. Time evolution of the secondary vortices at $R e=200$. The same vortices that appear in different snapshots are labelled with the same numbers. The labels above and below the vortices correspond to the negative and positive vortices, respectively. The symbols + and \& denote merging of the two-layered and secondary vortices, respectively.

As the secondary vortices propagated downstream, an interesting phenomenon, observed in Fig. 5(b,c), was that further merging of some of the same-sign secondary vortices and cancellation of some of the opposite-sign secondary vortices appeared at $x / D \gtrsim 200$. For example, in Fig. 5(b), positive secondary vortices 1 and 2 had just merged into a positive vortex $1 \& 2$ (where \& denotes merging of the secondary vortices), while at the same streamwise location negative secondary vortices 1 and $2+3+4$ were about to merge. Similar merging behaviours are also observed in Fig. 5(b) at $x / D \sim 230$ and 260 and in Fig. 5(c) at $x / D \sim 265$, where merging was observed for both positive and negative secondary vortices. Vortex cancellation is observed in Fig. 
5(c) at $x / D \sim 210$, where positive secondary vortex 52 and its negative counterpart $52+53$ had almost cancelled each other at this time instant. The merging and cancellation of the secondary vortices resulted in a gradual reduction of the number of secondary vortices with an increase of the distance downstream.

As shown in Fig. 5, the spatial arrangement of the secondary vortex street was highly irregular after its emergence (immediately after the irregular vortex merging process), and was complicated further by the propagation of the secondary vortices with the flow and in particular the further merging and cancellation of some of the secondary vortices at $x / D \gtrsim 200$. Nevertheless, there was no sign of a further transition at any specific streamwise locations after the transition to the secondary vortex street at $x / D \sim 77-110$, which was different from the speculation of Taneda (1959), who proposed that there was an alternate formation and breakdown of the secondary vortex street with distance downstream. Based on the visualisation of an instantaneous flow field (instead of the time evolution of the vortices), one may suspect that there is an alternate formation and breakdown of the secondary vortex street with distance downstream, e.g. in Fig. 5(c) the range of $x / D \sim 230-270$ looks like the breakdown of a regular secondary vortex street, while the range of $x / D \sim 280$ - 320 looks like the reappearance of a regular secondary vortex street. However, these far-wake patterns did not constitute transitions because (i) the relatively regular pattern at $x / D \sim 280-320$ was shaped at the transition to the secondary vortex street and was then simply advected downstream; and (ii) the further vortex merging behaviours that induced the breakdown of a regular secondary vortex street at e.g. $x / D$ $\sim 230-270$ occurred randomly for only a few vortices and developed very slowly (over a streamwise distance of more than 100D), instead of constantly occurring at particular streamwise locations (such as the transitions to the two-layered and the secondary vortex streets).

\subsection{Formation mechanism 1 (FM1) for $R e=160$}

The time evolution of the vortices at $R e=160$ was completely different from that at $R e=200$. Detailed time evolution of the vortices at $R e=160$ was examined for a time period of $t^{*}=2500-3370$, and is illustrated in Fig. 6 with the instantaneous vorticity field at a typical time instant $t^{*}=3000$. For $R e=160$, the two-layered vortices were annihilated into two parallel shear layers with no vortices at $x / D \sim 130$ (Fig. 6a), which was different from the case at $R e=200$ where the two-layered vortices merged and resulted in the secondary vortex street. It was also found that individual vortices would always reoccupy the entire parallel shear layers at locations further downstream of $x / D \gtrsim 320$ (e.g. Fig. 6c). The disappearance and reappearance of the vortices in the parallel shear layers indicated that the reappeared vortices certainly did not arise from the merging of the two-layered vortices. Instead, the hydrodynamic instability of the parallel shear layers induced flapping/waviness of the bare shear layers (i.e. shear layers with no vortices), which eventually triggered the reappearance of the vortices in the shear layers. The reappeared vortices did not emerge at a particular streamwise location. Instead, the reappearance spanned a long 
range of $x / D \sim 140-320$. For example, in Fig. 6(b) the parallel shear layers were only partly occupied by the reappeared vortices at $x / D \sim 190-205$, while the bare shear layers at $x / D \sim 175-190$ remained in this pattern for approximately another $50 D$ before vortices reappeared.

(a)

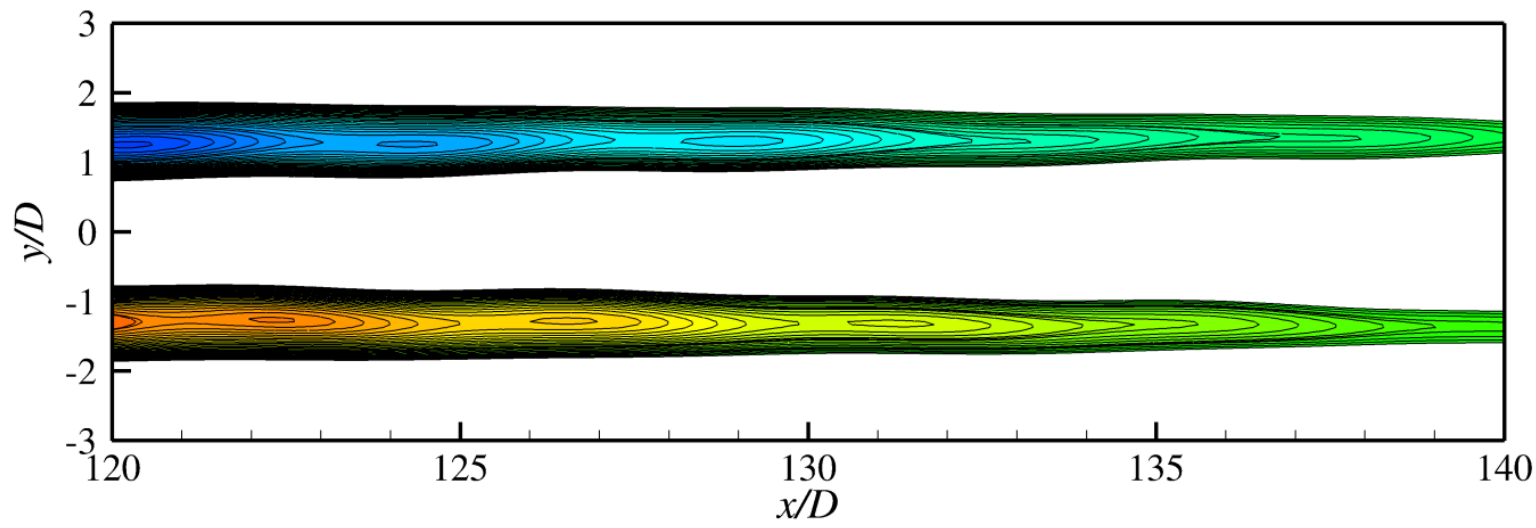

(b)

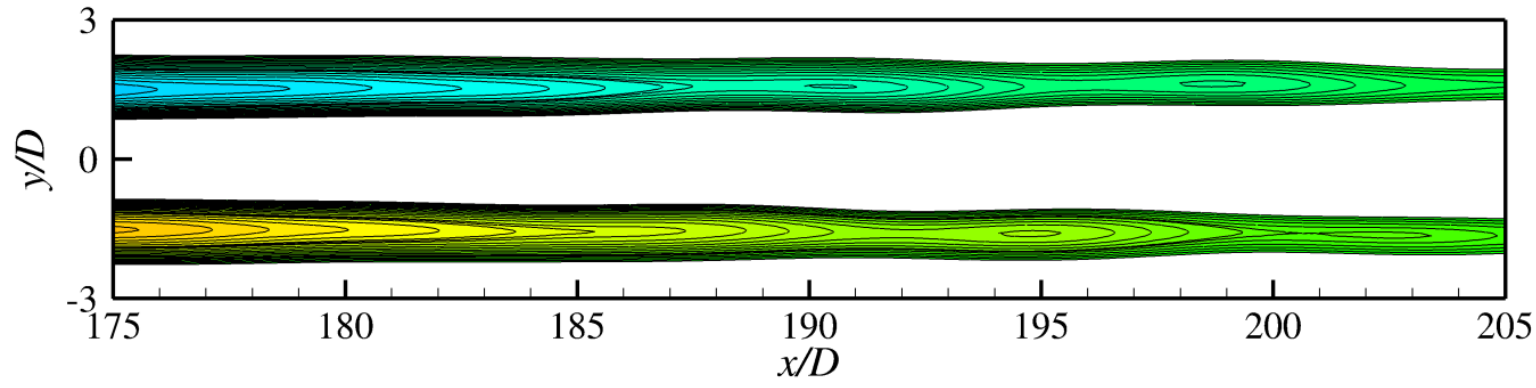

(c)

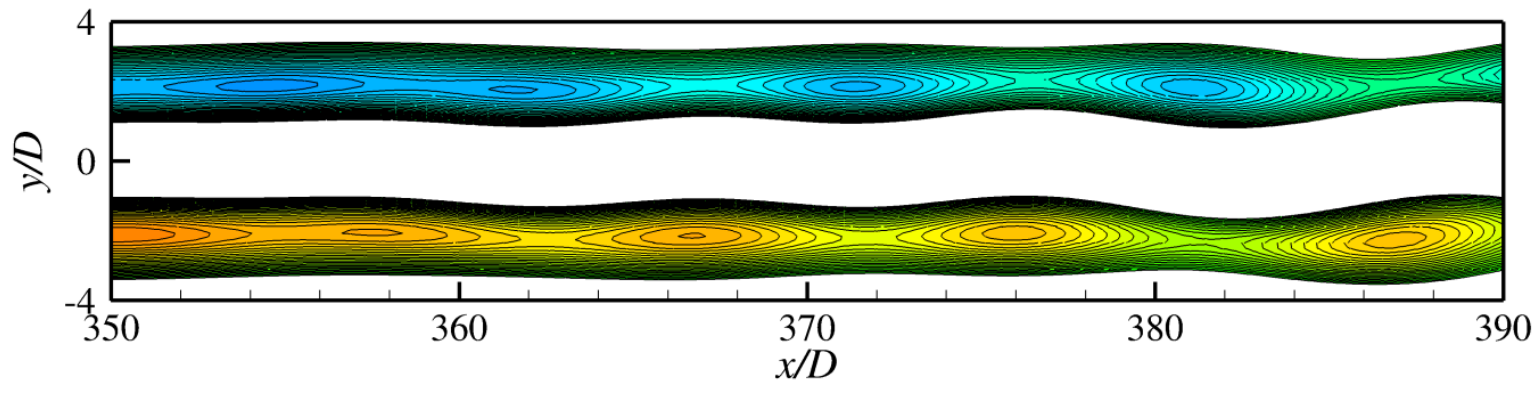

Fig. 6. Instantaneous vorticity field for $R e=160$ at a typical time instant of $t^{*}=3000$. The vorticity field is shown for (a) $x / D=120-140$ with $|\omega|=0.11-0.14$, (b) $x / D=$ $175-205$ with $|\omega|=0.07-0.10$, and (c) $x / D=350-390$ with $|\omega|=0.03-0.045$.

Compared with the secondary vortices of $R e=200$ shown in Fig. 5, the reappeared vortices at $R e=160$ (e.g. Fig. 6c) were much weaker in strength, such that they largely remained in the two shear layers. Fig. 7(a) shows the spatial distribution of the vortex centres for $R e=160$ at $t^{*}=3000$. After the transition to the two-layered vortex street at $x / D \sim 30$, the positive and negative vortices followed two clear linear pathways (i.e. the two shear layers) up to the downstream extent of the computational domain, which indicated that the reappeared vortices stayed in the two shear layers. This was in contrast to the irregular spatial distribution of the vortices at $R e=200$ (Fig. 5). 
(a)

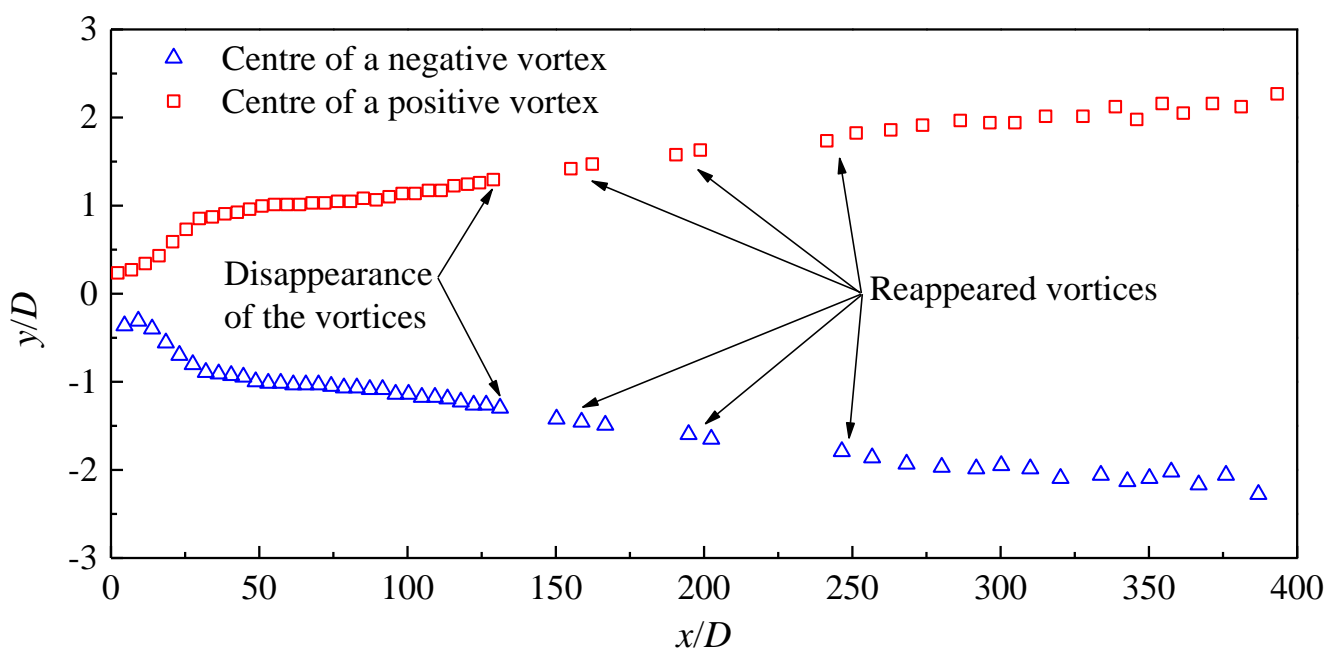

(b)

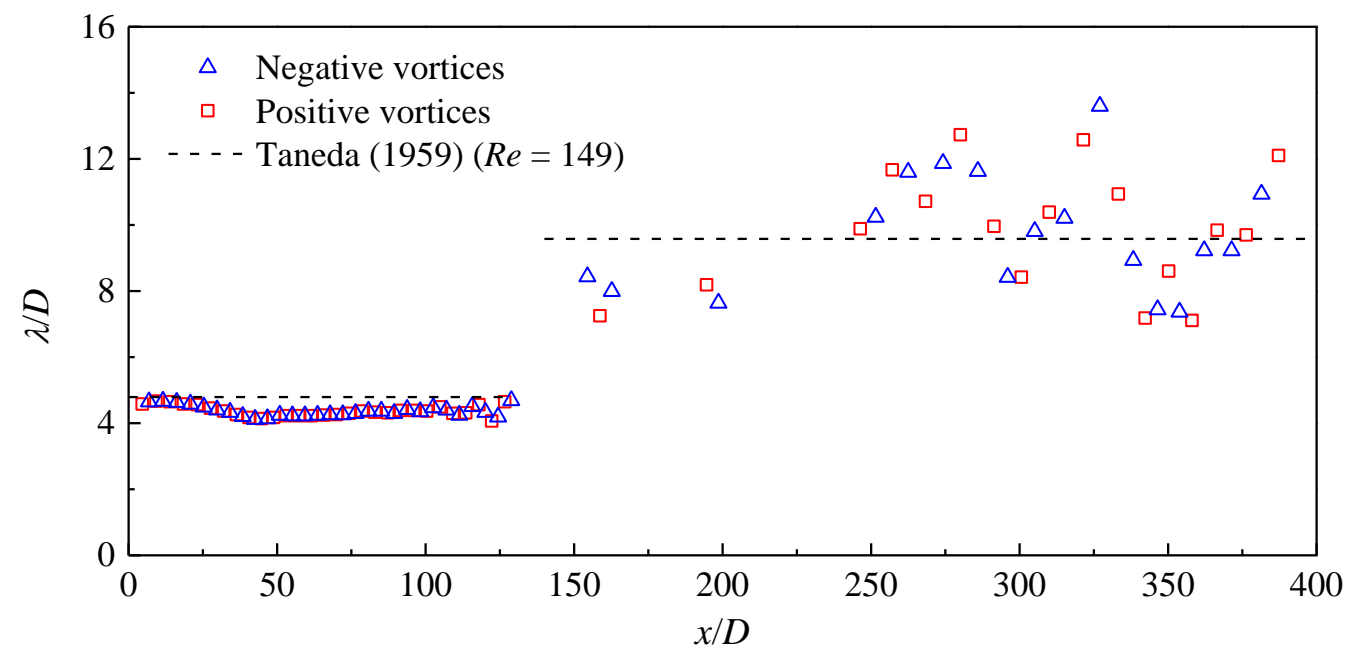

Fig. 7. Vortex patterns for $R e=160$ at the time instant $t^{*}=3000$. (a) Spatial distribution of the vortex centres, and (b) evolution of the spatial scale of the vortices with distance downstream.

Nevertheless, the spatial scale of the reappeared vortices was larger than that of the primary and two-layered vortices. The spatial scale of the vortices was quantified in Fig. 7(b) by the streamwise distance between the centres of the two adjacent same-sign vortices (denoted $\lambda$ ). As shown in Fig. 7(b), the $\lambda / D$ values for the reappeared vortices were roughly twice those of the primary and two-layered vortices. Fig. 7(b) also shows the $\lambda / D$ values for the primary and secondary vortices observed experimentally by Taneda (1959) at a similar $R e$ of 149 , whose results, although not detailing the fluctuations in $\lambda / D$ for the secondary vortices, were largely consistent with the present results. To be consistent with Taneda (1959), who found that the main feature of the secondary vortices is a spatial scale larger than the primary vortices, in the present study the reappeared vortices observed at $R e=160$ were considered as secondary vortices, although they were relatively weak and remained in the two shear layers. 


\subsection{Both FM1 and FM2 for intermediate Re of $170-180$}

Detailed time evolution of the vortices at $R e=170$ was examined for a time period of $t^{*}=2400-2800$. In general, the two-layered vortices were annihilated into two bare shear layers at $x / D \sim 120$, which was similar to that shown in section 3.2 for $R e=160$. An exception was that for a short time period of $t^{*}=\left(2400+54 T^{*}\right)-(2400$ $+62 T^{*}$ ), vortex merging similar to that of $R e=200$ (section 3.1) was observed over a range of $x / D \sim 100-120$ (see Fig. 8a). The vortices shown in Fig. 8(a) included four positive and four negative merging cycles as well as a negative single vortex (i.e. negative vortex 5). The merged vortices were not annihilated because their spatial scales were enlarged. In addition, the spatial scale of the single vortex 5 was also enlarged by the waviness of the shear layers induced by the vortex merging at the two sides of this vortex. These enlarged vortices were clearly identified as the secondary vortices as they propagated further downstream (Fig. 8b-d). The waviness of the two shear layers at the two sides of the group of the secondary vortices excited additional vortices, for example positive vortex 9 and negative vortex 10 shown in Fig. 8(c). However, the relatively weak vortices in the range of $x / D \sim 250-350$ in Fig. 8(c) that remained in the two shear layers were similar to those observed at $R e=160$ (see e.g. Fig. 6c) and were excited by the instability and flapping of the bare shear layers. In Fig. 8(d), as the group of the secondary vortices propagated further downstream, more vortices were excited at its two sides, which arose from the combined effects of (i) the increased waviness of the two shear layers induced by the further enlarged secondary vortices as they propagated downstream; and (ii) intrinsic instability of the shear layers.

(a)

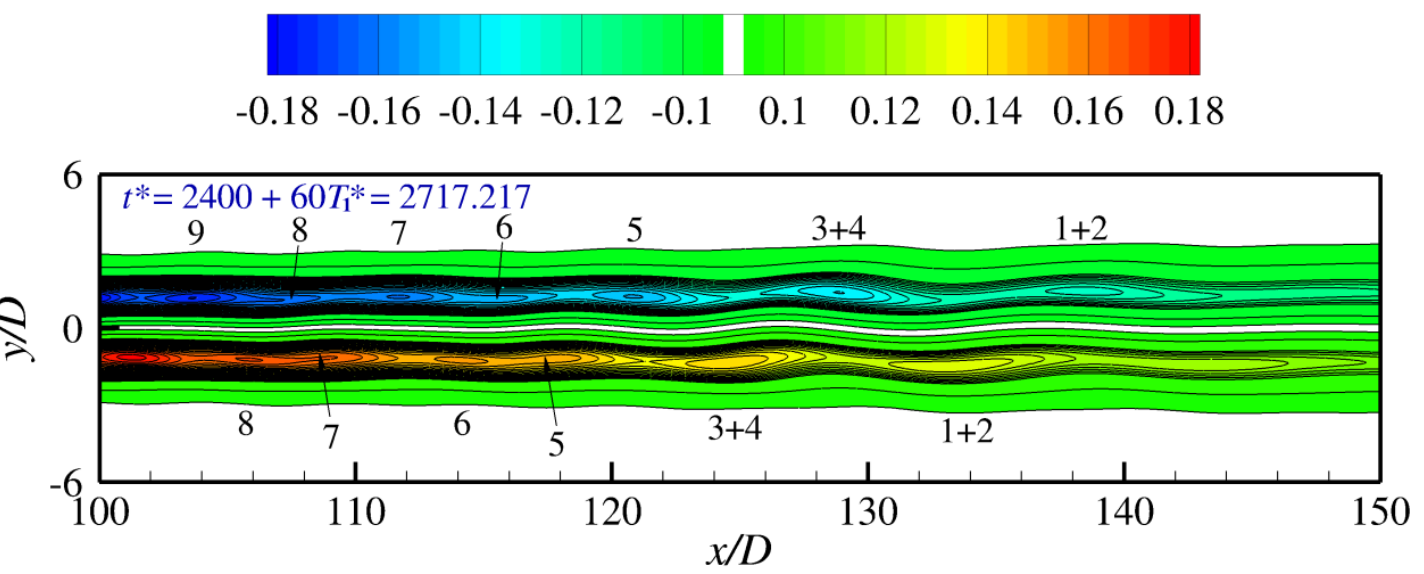


(b)

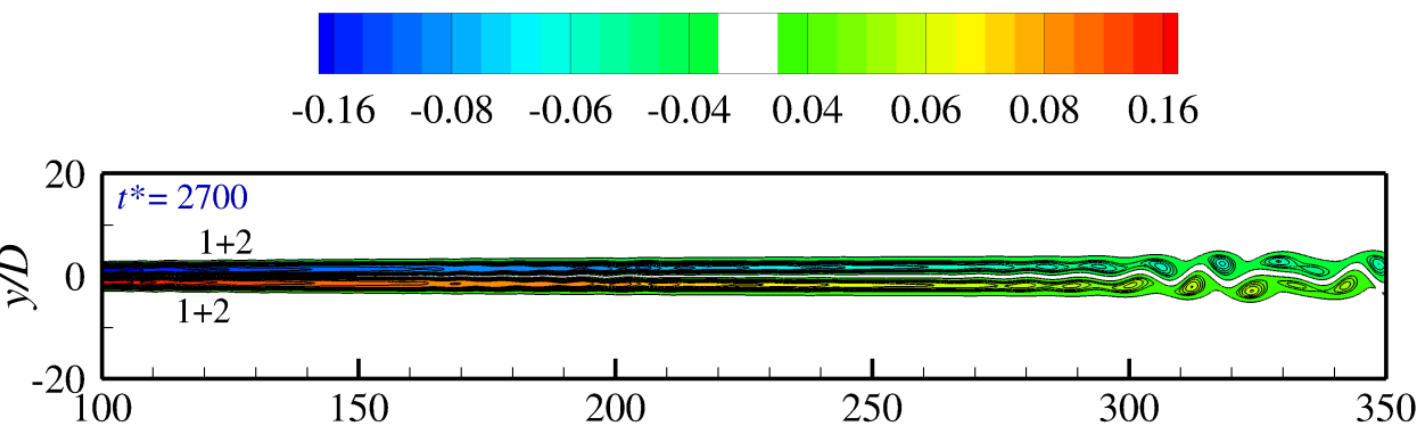

(c)

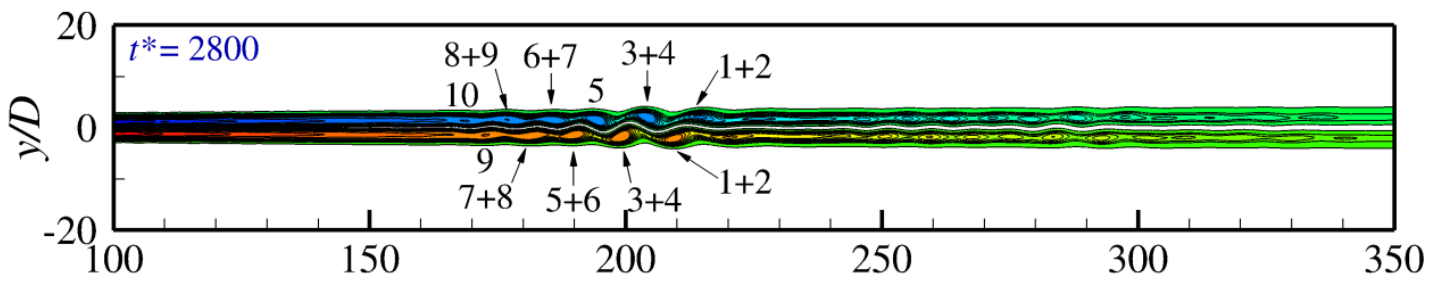

(d)

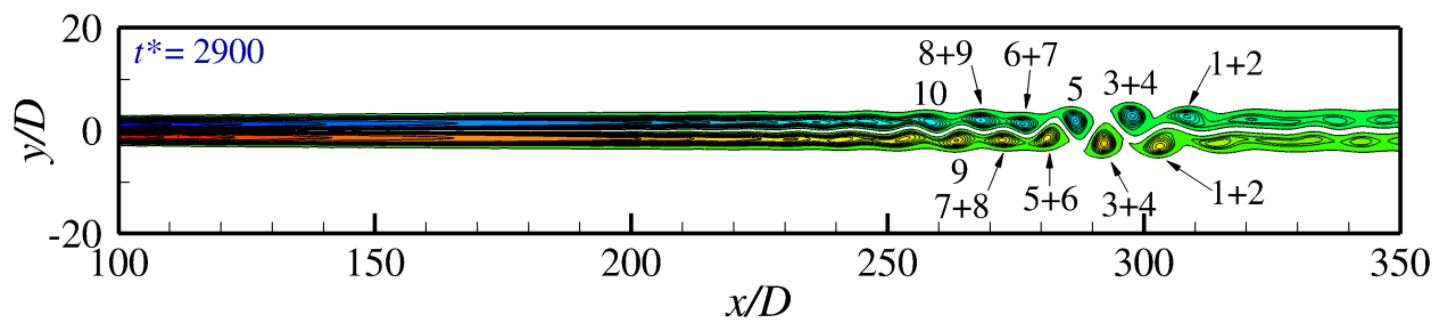

Fig. 8. Time evolution of the secondary vortices at $R e=170$. The same vortices that appear in different snapshots are labelled with the same numbers. The labels above and below the vortices correspond to the negative and positive vortices, respectively.

The detailed time evolution of the vortices at $R e=180$ was examined for time periods of $t^{*}=2000-2360$ and $2900-3100$. In general, the two-layered vortices merged into secondary vortices over a range of $x / D \sim 90-110$, which was similar to that shown in section 3.1 for $R e=200$. An exception was that for a short time period of $t^{*}=\left(2900+13 T^{*}\right)-\left(2900+21 T^{*}\right)$, the two-layered vortices were annihilated into two bare shear layers at $x / D \sim 113$, which was similar to that shown in section 3.2 for $R e=160$. New vortices were excited from these fractions of bare shear layers downstream at $x / D \sim 115-140$.

\section{Physical mechanism controlling the manifestation of FM1 and FM2}

Section 3 provides clear evidence that both FM1 and FM2 are at play for the wake transition to the secondary vortex street. At $R e=200$ (section 3.1), the secondary vortices were formed from the merging of the same-sign vortices in the two-layered vortex street (i.e. FM2). In contrast, at $R e=160$ (section 3.2) and similarly at $R e=150$, the secondary vortices were the reappeared vortices arising from the flapping of the bare shear layers at streamwise locations where the two-layered vortices had already been annihilated, owing to the hydrodynamic instability of the shear layers (i.e. FM1). At $R e=160$, the flapping of the bare shear layers induced secondary vortices at $x / D \sim 140-320$, while at $\operatorname{Re}=150$, secondary 
vortices were observed at more downstream locations of $x / D \gtrsim 280$. At $R e \leq 140$, secondary vortices were not observed within the effective computational domain length up to $x / D=400$.

In addition, to the best of our knowledge, it was observed for the first time that for an individual case (e.g. at $R e=170$ or 180 ), the secondary vortices can be formed from both FM1 and FM2. As $R e$ was increased from 170 to 180, the main formation mechanism changed from FM1 to FM2. Therefore, as Re increases from 160 to 200, the probability of the occurrence of FM1 gradually reduces from $100 \%$ to $0 \%$, whereas that of FM2 increases from $0 \%$ to $100 \%$. The gradual transition of the formation mechanism with $R e$ (rather than an abrupt switchover at a particular $R e$ ) suggested that the two formation mechanisms were interchangeable, with their probabilities of occurrence dictated by an intrinsic factor.

This intrinsic factor, which controls the manifestation of either FM1 or FM2, was investigated as follows. Fig. 9 summarises the ranges of $x / D$ for the emergence of the secondary vortices (the shaded area). The secondary vortices formed from FM1 and FM2 are distinguished by blue and red vertical bars, respectively. At intermediate $R e$ values of 170 and 180, the secondary vortices were formed from both mechanisms in an alternate manner, and both red and blue vertical bars existed. It was found that the red and blue vertical bars were separated at the $x / D$ value corresponding to the annihilation of the two-layered vortices (determined at the time periods when the secondary vortices were formed from FM1). Therefore, whether the secondary vortices were formed from FM1 or FM2 was dictated by the streamwise location for the annihilation of the two-layered vortices (the black curve in Fig. 9) relative to the streamwise locations for the emergence of the secondary vortices (the shaded area in Fig. 9). Secondary vortices emerging prior to the possible annihilation of the two-layered vortices were formed from FM2, where the transition made direct use of the two-layered vortices at hand. In contrast, secondary vortices emerging downstream of the annihilation of the two-layered vortices were formed from FM1, where the transition created new vortices through flapping of the bare shear layers. 
$\longrightarrow$ Secondary vortices formed from FM1

$\rightarrow$ Secondary vortices formed from FM2

- Annihilation of the two-layered vortices

- - - Extension of the curve of vortex annihilation based on the modified case

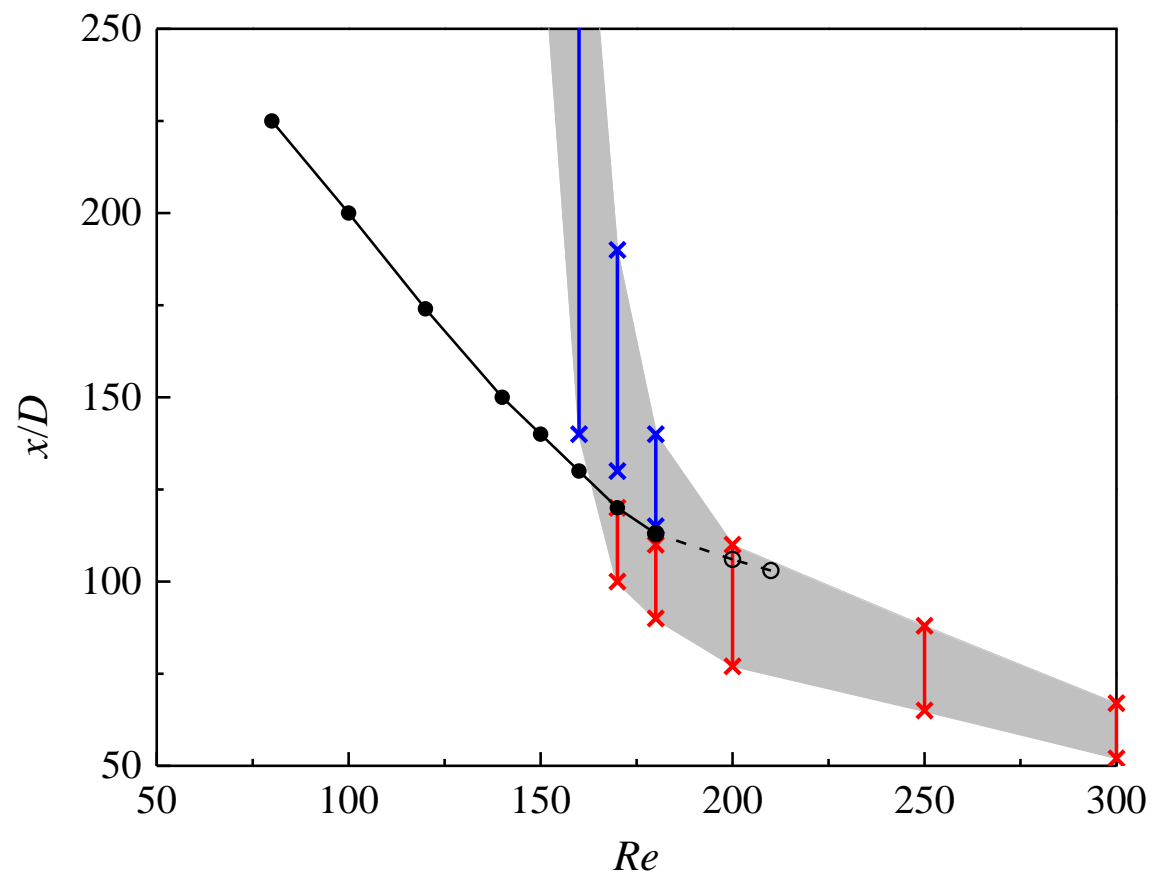

Fig. 9. Ranges of $x / D$ where the secondary vortices are formed from either FM1 or FM2. The entire range of $x / D$ for the emergence of the secondary vortices is shaded. The numerical results of $R e=250$ and 300 in the extended laminar regime are reproduced from Jiang and Cheng (2019).

For $R e \geq 200$, the streamwise location for the annihilation of the two-layered vortices was unable to be revealed by the natural flow, because the secondary vortices were formed from FM2 only (prior to any possible annihilation of the two-layered vortices). To reveal the streamwise location for the vortex annihilation, the case of $R e$ $=200$ was simulated with a modified computational mesh that included an artificial horizontal slip plate of zero-thickness placed only at the wake centreline and at $x / D \geq$ 80. The slip boundary condition included $\partial u_{x} / \partial y=0, u_{y}=0$ and a high-order Neumann condition for the pressure. The slip plate did not affect the transition to the two-layered vortex street at $x / D \sim 28.7$ but eliminated potential interactions between the two parallel rows of vortices (and thus the vortex merging process) that could have occurred at $x / D \geq 80$. By eliminating vortex merging, the modified case showed that the two-layered vortices would be annihilated at $x / D \sim 106$, as shown by an open circle in Fig. 9. This point was slightly smaller than the largest $x / D$ for the vortex merging, owing to the existence of successive merging of three vortices (see section 3.1).

However, a similar modified case at $R e=210$ showed strong interactions between the two parallel rows of vortices prior to the slip plate at $x / D \geq 80$, which was likely to be because vortex merging occurred earlier at a larger $R e$ (Fig. 9). Therefore, for the 
modified case at $R e=210$, a slip plate of $x / D \geq 70$ was used. The extension of the $x / D-R e$ relationship for the vortex annihilation (the dashed curve with open circles in Fig. 9) ended at $R e=210$ because a similar modified case at $R e=220$ showed that even when the slip plate for $x / D \geq 70$ was in use, each row of vortices could still merge and result in the secondary vortices on its own (without vortex annihilation).

The extension of the $x / D-R e$ relationship for the vortex annihilation suggested that this curve had a tendency to cut the shaded area in Fig. 9 into two halves, with the upper and lower halves corresponding to FM1 and FM2, respectively. This finding explains why both formation mechanisms can exist for an individual case (e.g. at $R e=$ 170 or 180). The reason was that the streamwise location for the emergence of the secondary vortices moves back and forth over time, i.e. the secondary vortices emerge over a range of $x / D$ rather than at a single $x / D$ for each $R e$, such that when this range of $x / D$ is cut through by the curve of vortex annihilation shown in Fig. 9, two mechanisms exist for the same case. In contrast, for some other bluff-body wakes, where the secondary vortices emerge at a fixed $x / D$ for each $R e$, FM1 and FM2 cannot co-exist in any individual case.

The present finding also explains why some previous studies identified only FM1 or FM2.

(i) Cimbala et al. (1988), Williamson and Prasad (1993) and Kumar and Mittal (2012) attributed the emergence of the secondary vortices to FM1, which, according to Fig. 9, is because these studies investigated the wake of a circular cylinder at $R e \sim 150$, where the secondary vortices emerge downstream of the annihilation of the two-layered vortices.

(ii) Inoue and Yamazaki (1999) suggested that the existence of free-stream frequency forcing was the reason why the formation mechanism changed from FM1 to FM2. However, based on the vorticity fields shown by Inoue and Yamazaki (1999), another important effect of free-stream forcing is an earlier emergence of the secondary vortices in the wake. For example, for unforced and forced wakes of a circular cylinder at $R e=150$, the secondary vortices emerge at $x / D \gtrsim 150$ and 60, respectively (Inoue and Yamazaki, 1999). Therefore, it is believed that the significant upstream movement of the location for the emergence of the secondary vortices, specifically from downstream to well upstream of the location for the annihilation of the two-layered vortices ( $x / D=140$ in Fig. 9), is the real reason for the change of the formation mechanism from FM1 to FM2.

(iii) The present finding is also applicable to bluff bodies other than a circular cylinder. For example, FM2 was identified in the wake of an elliptical cylinder with $R e=150$ and $A R=0.25$ (Thompson et al., 2014), as well as in the wake of tandem circular cylinders with $R e=100$ and a cylinder centre-to-centre distance of $8 D$ (Wang et al., 2010). This is because for these scenarios the secondary vortices emerge relatively close to the cylinder (within approximately $20 D$ downstream of the cylinder), prior to the possible annihilation of the two-layered vortices. However, FM1 was identified in the wake of a rectangular cylinder with $R e=80-100$ and $A R$ 
$=0.2$ (Mizushima et al., 2014), since for this scenario the secondary vortices emerge downstream of the annihilation of the two-layered vortices.

\section{The $R e$-dependence of the emergence of the secondary vortices}

As shown earlier in Fig. 9, the manifestation of either FM1 or FM2 is governed by the $x / D-R e$ relationship for the annihilation of the two-layered vortices and the $x / D-R e$ relationship for the emergence of the secondary vortices. The latter relationship indicates that with increasing $R e$ the secondary vortices emerge at decreasing $x / D$ and over a decreasing range of $x / D$. The $R e$-dependence of the emergence of the secondary vortices was investigated further in this section by exploring the fundamental cause for the emergence of the secondary vortices.

\subsection{Characteristics of the shear layers in the intermediate wake}

Kumar and Mittal (2012) investigated the intermediate and far wake of $R e=150$ and characterised the hydrodynamic instability in FM1 as a convective instability. This study will show that convective instability is responsible for the emergence of the secondary vortices for all $R e$ values, regardless of its manifestation through FM1 or FM2.

It is well known that the rearrangement of the vortices from the primary vortex street to the two-layered vortex street would divert the flow away from the wake centreline and result in a "calm region" near the wake centreline (in between the two layers of vortices) where the streamwise velocity is relatively small (Durgin and Karlsson, 1971). The present results showed that the transition to the two-layered vortex street occurred at around $x / D=26-29$ (corresponding to local maxima of $u_{y}$ ) for $R e=140-200$, and correspondingly the $u_{x}$ value sampled along the wake centreline decreased over $x / D \sim 20-40$ (Fig. 10a). Fig. 10(a) also shows that with increasing $R e$ the velocity deficit at the wake centreline increased. Fig. 10(b) illustrates the $u_{x}$ profiles sampled at $x / D=40$, which showed that with increasing $R e$ the velocity deficit increased while the wake width decreased, and both contributed to an increase in the shear rate of the two shear layers. Fig. 10(c) quantifies the maximum shear rate $\left(\partial u_{x} / \partial y\right)_{\max }$ of the $u_{x}$ profiles sampled at various $x / D$ locations for various $R e$ values. With the increase in $R e$, the maximum shear rate gradually increased, which suggested that the shear layers became increasingly convectively unstable to allow for an earlier (in terms of the streamwise location) emergence of the secondary vortices. The convective instability of the shear flow for the present case was similar to the well-known Kelvin-Helmholtz instability for the plane mixing layer (e.g. Drazin, 1970; Brown and Roshko, 1974; Rogers and Moser, 1992) and the separating shear layer of a cylinder (e.g. Prasad and Williamson, 1997). 
(a)

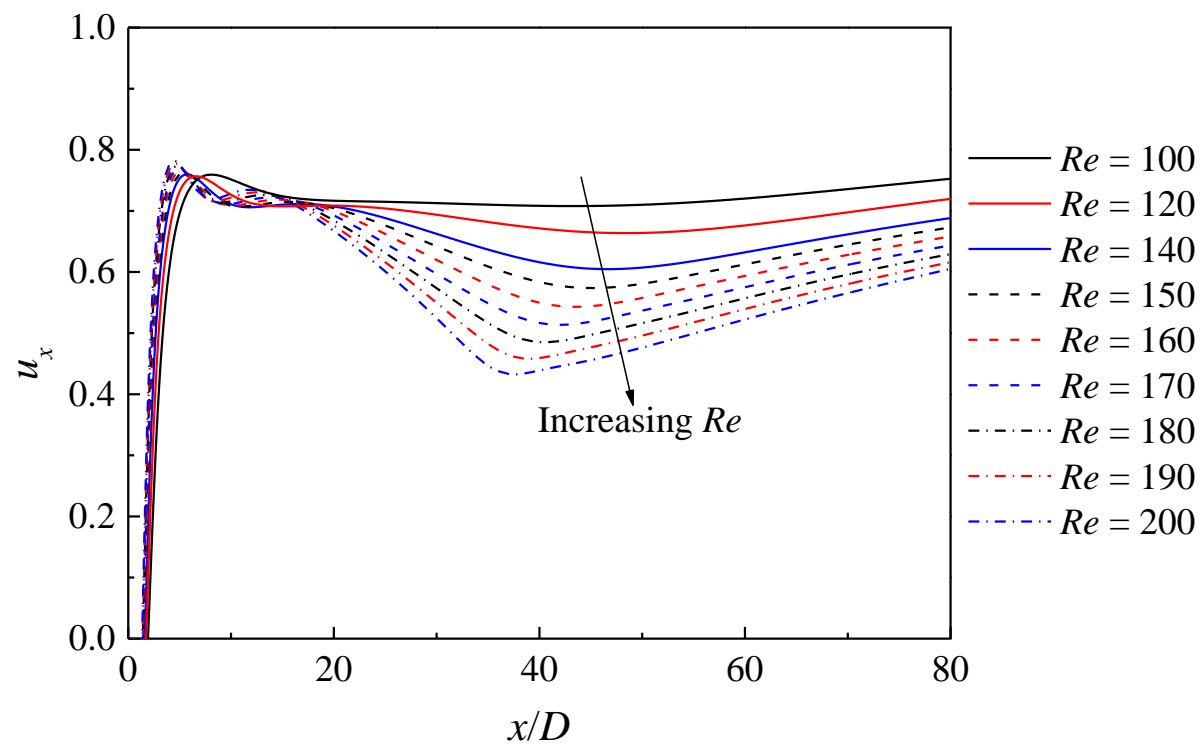

(b)

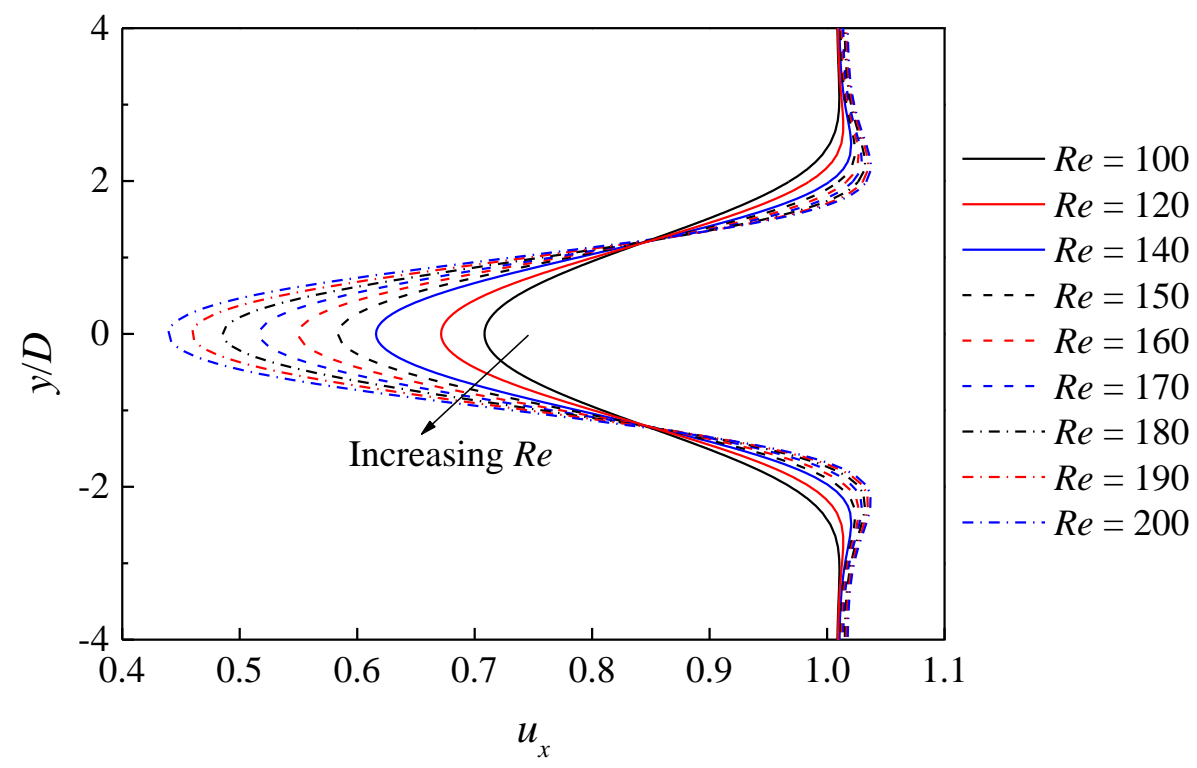

(c)

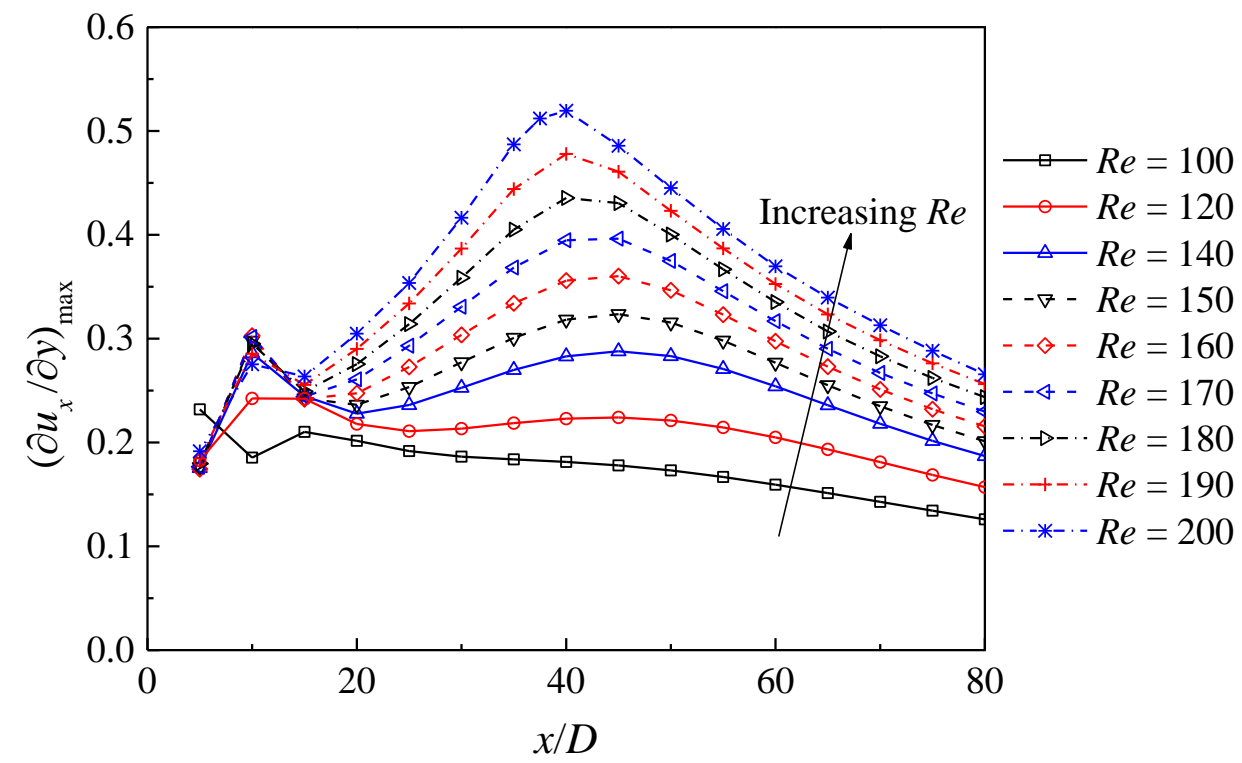

Fig. 10. Characteristics of the $u_{x}$ profiles for various $R e$ values: (a) The $u_{x}$ profiles 
sampled along the wake centreline, (b) the $u_{x}$ profiles sampled at $x / D=40$, and (c) the maximum shear rate of the $u_{x}$ profiles sampled at various $x / D$ locations.

\subsection{Transient growth analysis of the intermediate wake}

To investigate the $R e$-dependence of the shear-layer characteristics in the intermediate wake (which is responsible for the $R e$-dependence of the emergence of the secondary vortices) more quantitatively, transient growth analysis was adopted in this section to quantify the maximum growth of the perturbation energy in the time-averaged wake. The transient growth analysis was performed for $R e=100-200$, which enabled an examination of the $R e$-dependence of the maximum growth. In addition, the range of $R e=100-200$ incorporates both FM1 and FM2, and this range will be examined if convective instability is shown to be the universal underlying mechanism.

Fig. 11 summarises the optimal energy growths $G(\tau)$ calculated under various combinations of $\tau$ and $R e$. As shown in Fig. 11, the transient energy growths for different $R e$ values shared similar qualitative trends, which suggested that there was no fundamental difference among the different $R e$ values. Therefore, the massive amplification of the perturbation energy was a prerequisite for the emergence of the secondary vortices for all $R e$ values, regardless of their manifestation through FM1 or FM2.

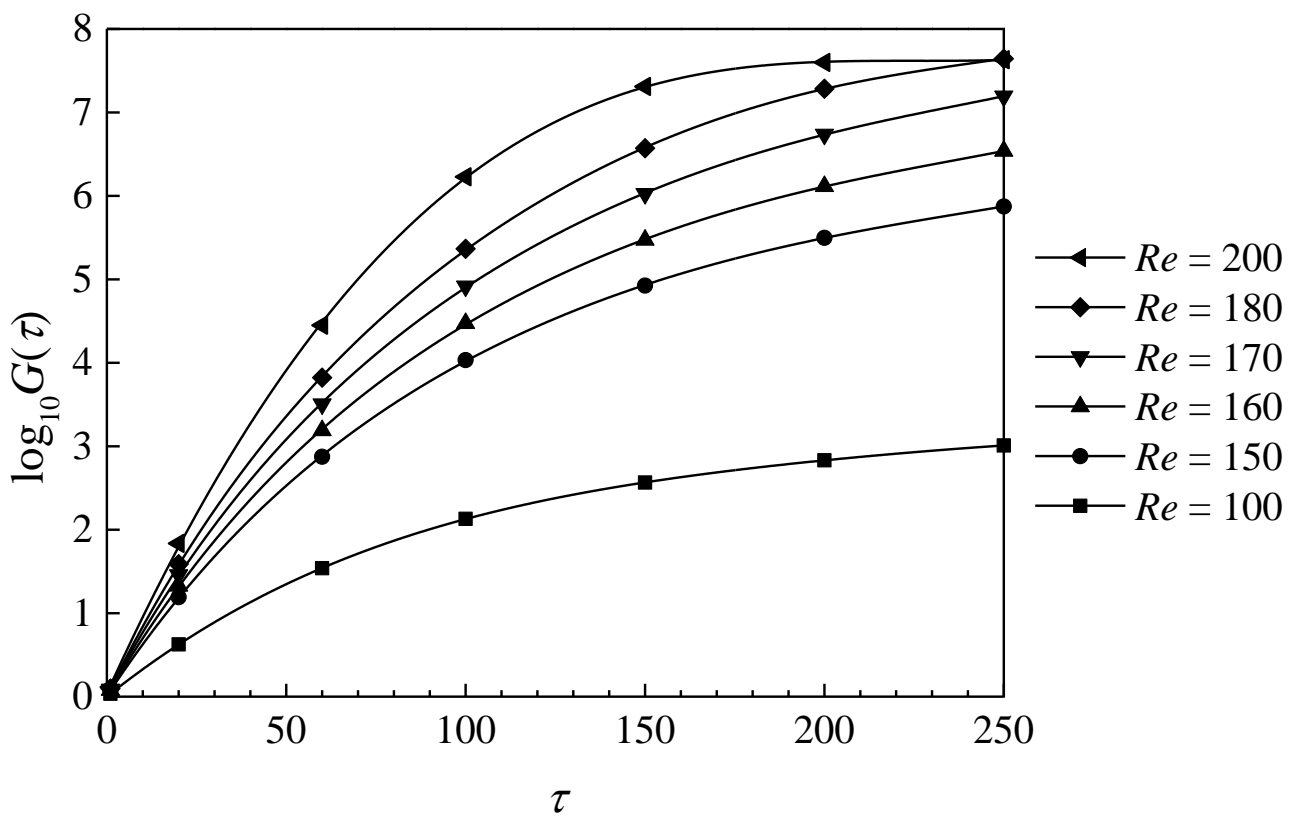

Fig. 11. Optimal energy growth $G(\tau)$ as a function of the time interval $\tau$ for various $R e$ values.

Fig. 12 illustrates further the results of $R e=180$ as an example, where the curve $G(\tau)-\tau$ is reproduced from Fig. 11. Also shown in Fig. 12 are the linear energy growths $E\left(t^{*}\right) / E(0)$ evolved from the optimal initial perturbations for various $\tau$ values. Based on an additional case with $\tau=60$ and a reduced domain size up to $x / D=300$, it 
was confirmed that the time evolution of the linear energy growth was unaffected by the location of the outlet boundary (until the perturbation was washed out of the domain). Naturally, each energy growth curve shown in Fig. 12 osculated the optimal growth curve $G(\tau)-\tau$ at $t^{*}=\tau$, i.e. the optimal growth curve was the envelope of the energy growth curves for various $\tau$ (Blackburn et al., 2008). In addition, the energy growth curves for relatively large $\tau$ values (e.g. $\tau=100$ and 200) corresponded approximately quantitatively to the envelope. Therefore, the energy growth for the case $\tau=100$ or 200 may serve as an approximation of the optimal energy growth in a time evolution manner.

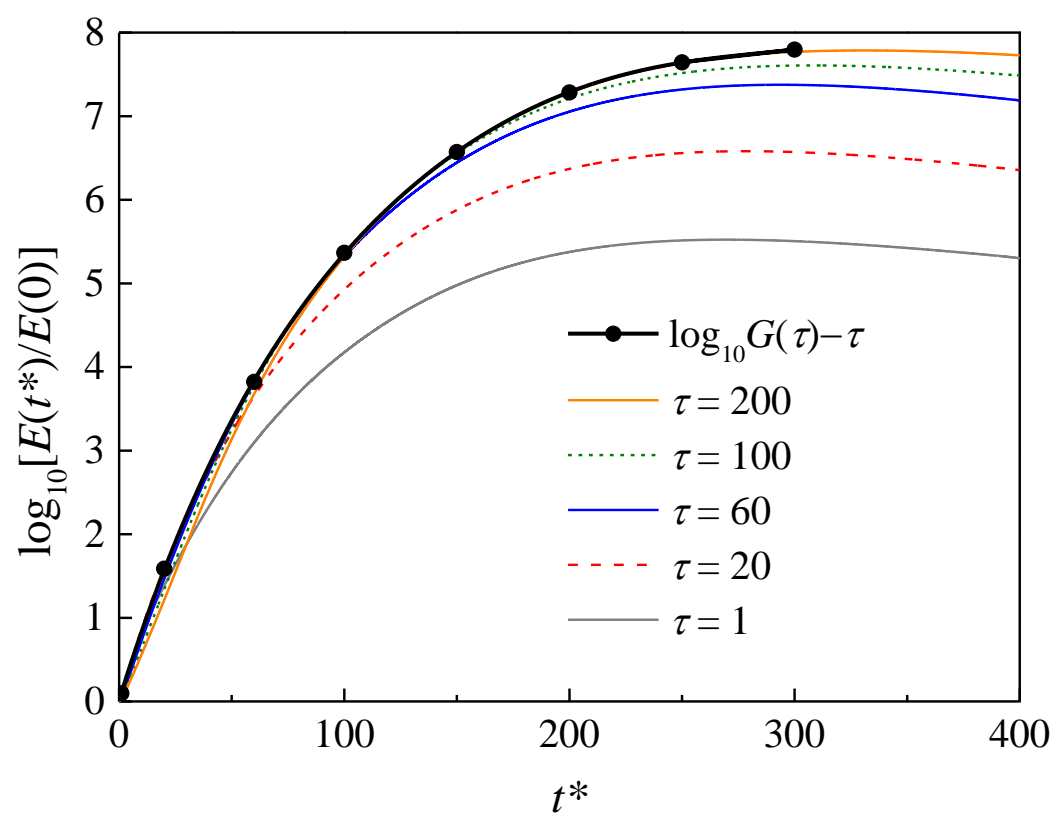

Fig. 12. Optimal energy growth curve $\log _{10} G(\tau)-\tau$ for $R e=180$, together with the linear energy growths $E\left(t^{*}\right) / E(0)$ evolved from the optimal initial perturbations for various $\tau$ values.

The energy growth for the case $\tau=100$ is further shown in Fig. 13 by the time evolution of the linear perturbation vorticity field. The case $\tau=100$ was chosen because it produced the optimal energy growth $G(\tau)$ at $t^{*}=\tau=100$ and corresponded to $x / D \sim 80-120$ (Fig. 13b), which was close to the range of $x / D$ for the emergence of the secondary vortices $(x / D \sim 90-140$ in Fig. 9). To show clearly the initial evolution of the perturbation, the vorticity fields in Fig. 13(a) were plotted with a vorticity range 100 times smaller than that used in Fig. 13(b). The time evolution of the linear perturbation vorticity field shown in Fig. 13 was consistent with the energy growth trend of $\tau=100$ shown in Fig. 12, where a massive energy amplification was observed for $t^{*}$ up to approximately 300 , followed by a gradual energy decay afterwards. This observation confirmed that the flow was globally stable but locally convectively unstable. 


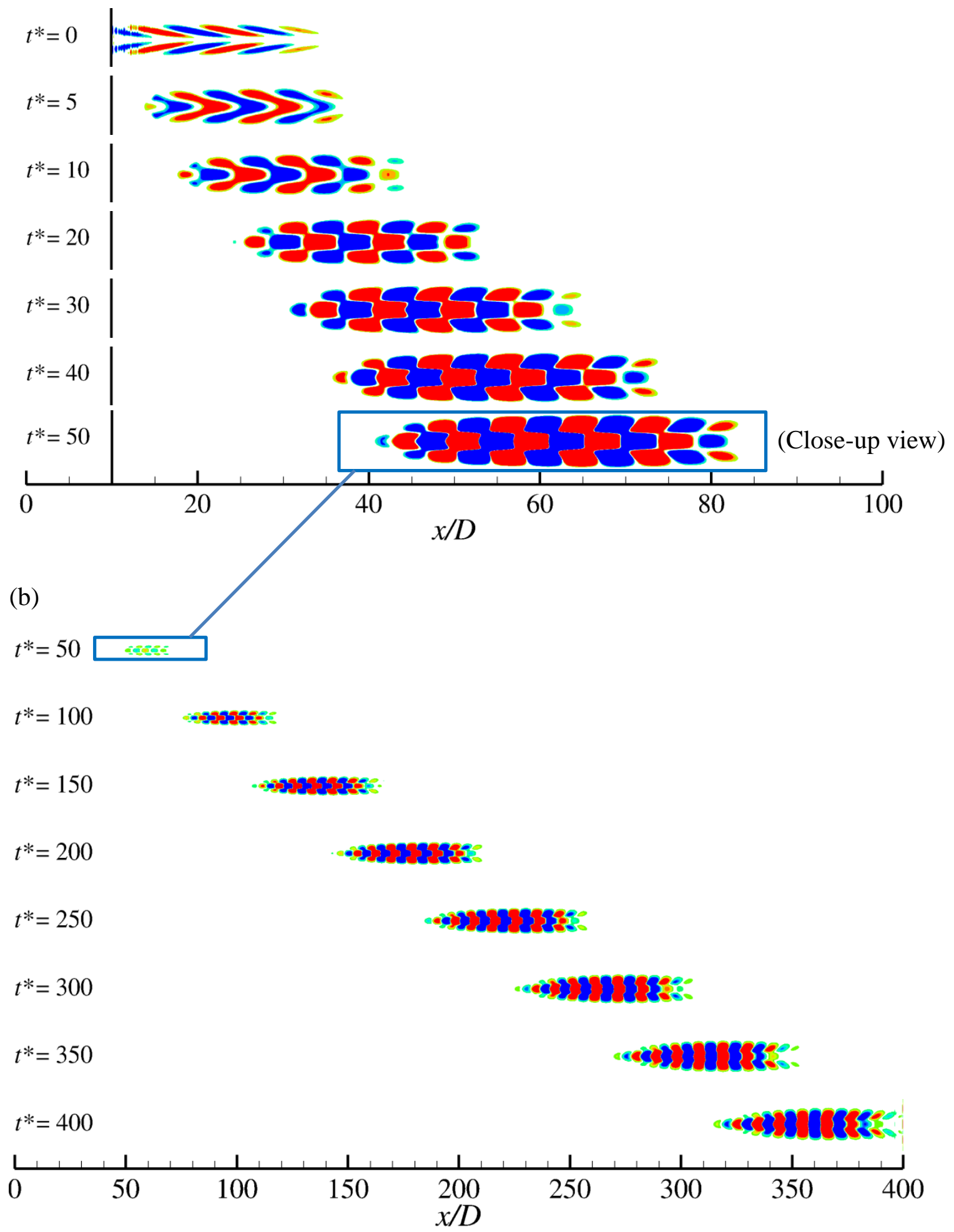

Fig. 13. Time evolution of the linear perturbation vorticity field developed from the optimal initial perturbation for $R e=180$ and $\tau=100$. The linear perturbation vorticity fields are symmetric about the wake centreline. Positive and negative vorticity values are in red and blue, respectively. The range of vorticity in panel (a) for $t^{*}=0-50$ is 100 times smaller than the range in panel (b) for $t^{*}=50-400$. In panel (a), the vertical line at $x / D=10$ represents the left end of the domain. 
It is interesting to note that the optimal initial perturbation shown in Fig. 13(a) (at $\left.t^{*}=0\right)$ shared close similarity to the two-dimensional optimal initial perturbations for plane Poiseuille, Couette and backward-facing step flows, where a series of highly strained counter-rotating rollers are observed and their inclination is opposite to the direction of the mean shear (Farrell, 1988; Butler and Farrell, 1992; Blackburn et al., 2008). The mean shear for the channel flows, such as the plane Poiseuille and backward-facing step flows, was in an opposite direction to that of the present wake flow, and, therefore, so was the inclination of the perturbation rollers. The fundamental similarity of the initial perturbation structures for all of these flows confirmed that the transient energy growth of the present wake flow also arose from the mean shear, as discussed earlier in section 5.1.

It was also seen in Fig. 13(a) that, with the evolution in time, the upper and lower rows of the counter-rotating rollers quickly interacted and formed an integrated pattern, which suggested interactions of the two shear layers. The integrated pattern stabilised at $t^{*} \gtrsim 20$ and then appeared similar to that shown by Kumar and Mittal (2012).

\subsection{Effect of the Reynolds number}

The $R e$-dependence of the optimal energy growth is quantified in Fig. 11, where $G(\tau)$ increased noticeably with the increase in $R e$. For example, an increase in $R e$ by 20 resulted in an increase in $G(150)$ of approximately one order of magnitude. The dependence of $G(\tau)$ on $R e$ suggested that the wake was increasingly convectively unstable at increasing $R e$ values. Consequently, with the increase in $R e$, the secondary vortices emerged at decreasing $x / D$ values (Fig. 9).

The effect of $G(\tau)$ on the $x / D$ for the emergence of the secondary vortices was further analysed as follows. To facilitate analysis of the time evolution of the perturbation, the optimal energy growth curve $G(\tau)-\tau$ for each $R e$, as shown in Fig. 11, was approximated by the linear energy growth curve $\left[E\left(t^{*}\right) / E(0)\right]-t^{*}$ evolved from the optimal initial perturbation for $\tau=200$ (Fig. 14), since the two curves are quantitatively similar (e.g. Fig. 12). To generate a link between the energy growth over $t^{*}$ and that over $x / D$, the streamwise advection of the perturbation for $\tau=200$ was quantified by the movement of the centroidal location of the perturbation energy $\left(x_{c} / D\right)$ over time (Fig. 15a), where $x_{c}$ is calculated as

$$
x_{c}=\frac{\int_{\Omega} \frac{1}{2}\left(u_{x}^{\prime 2}+u_{y}^{\prime 2}\right) x \mathrm{~d} \Omega}{\int_{\Omega} \frac{1}{2}\left(u_{x}^{\prime 2}+u_{y}^{\prime 2}\right) \mathrm{d} \Omega},
$$

In addition, Fig. 15(b) shows that the advection velocity of $x_{c} / D$ was not a constant. As illustrated by an example at $R e=150$, the variation of the advection velocity with distance downstream qualitatively followed the trend of the time-averaged streamwise velocity profile sampled along the wake centreline (while the quantitative difference in the velocity was because the velocity deficit at the wake centreline was the largest). 


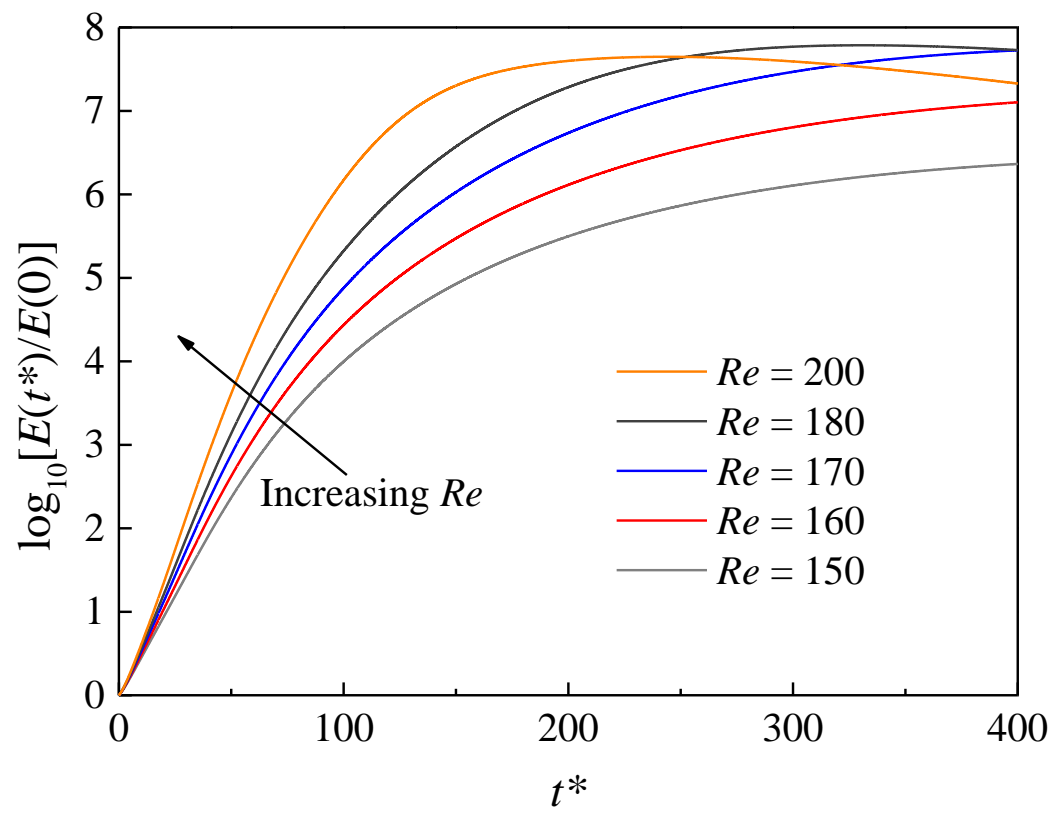

Fig. 14. Linear energy growth $E\left(t^{*}\right) / E(0)$ evolved from the optimal initial perturbation for $\tau=200$.

(a)

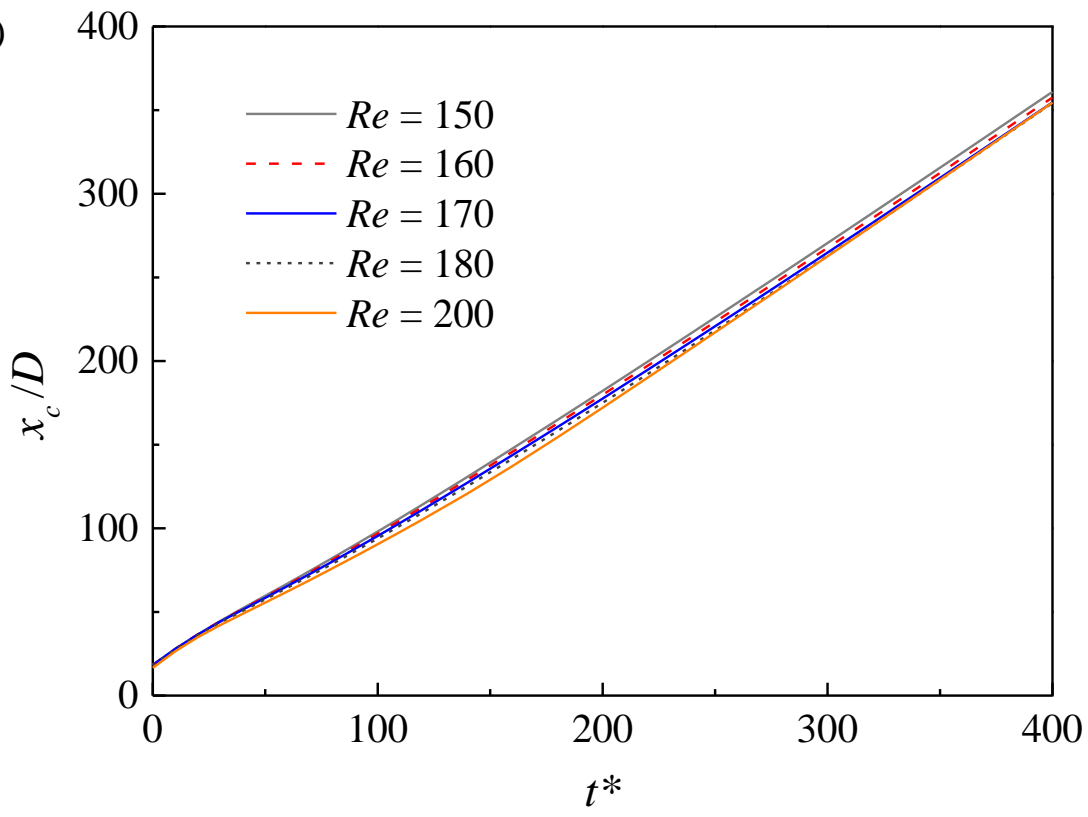


(b)

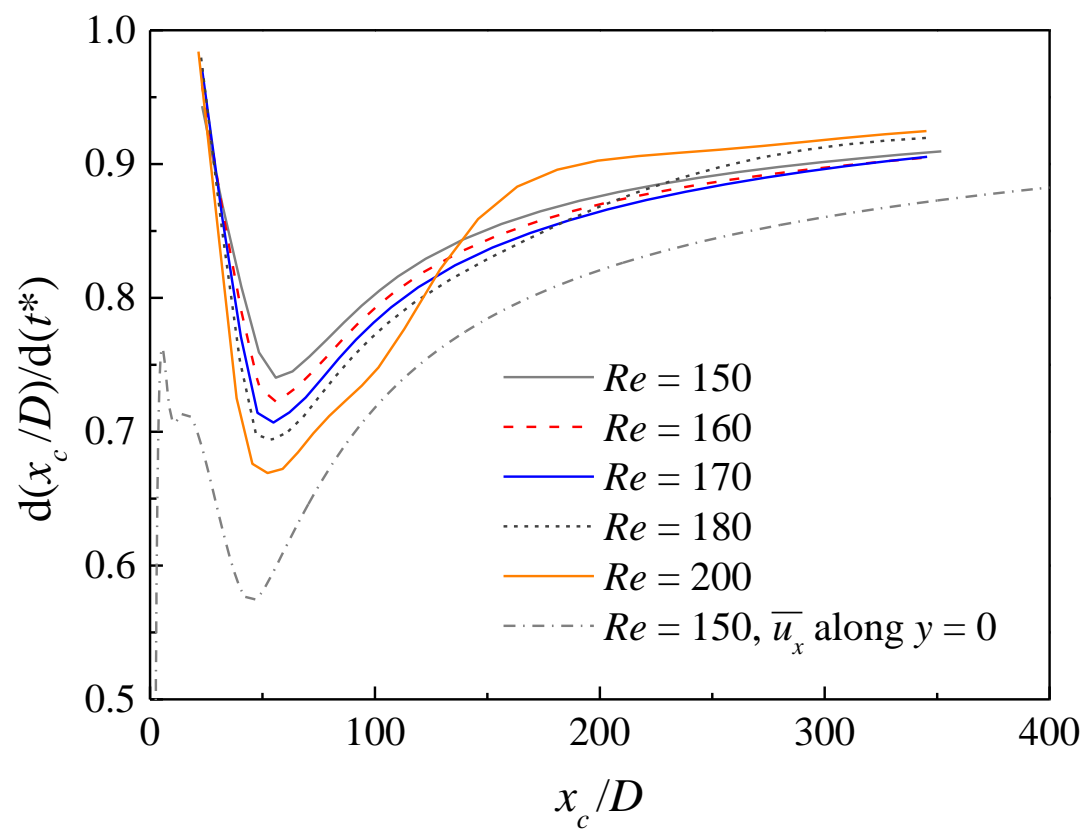

Fig. 15. Movement of the centroidal location of the perturbation energy over time for $\tau=200$ and various $R e$ : (a) the relationship between $x_{c} / D$ and $t^{*}$, and (b) the advection velocity of $x_{c} / D$ as a function of $x_{c} / D$.

Based on the relationship between $x_{c} / D$ and $t^{*}$ shown in Fig. 15(a), the linear energy growth curve $\left[E\left(t^{*}\right) / E(0)\right]-t^{*}$ shown in Fig. 14 was transformed into $\left[E\left(t^{*}\right) / E(0)\right]-\left(x_{c} / D\right)$ in Fig. 16. Fig. 16 also highlights (in the thick segment of the curve) the range of $x / D$ for the emergence of the secondary vortices (previously shown in Fig. 9). The secondary vortices generally emerged at energy amplification levels of $10^{5}-10^{7}$. This observation suggested that, with the increase in $R e$, the increasing gradient in the energy amplification was responsible for the emergence of the secondary vortices at decreasing $x / D$ and for a decreasing range of $x / D$, both of which were shown originally in Fig. 9. 


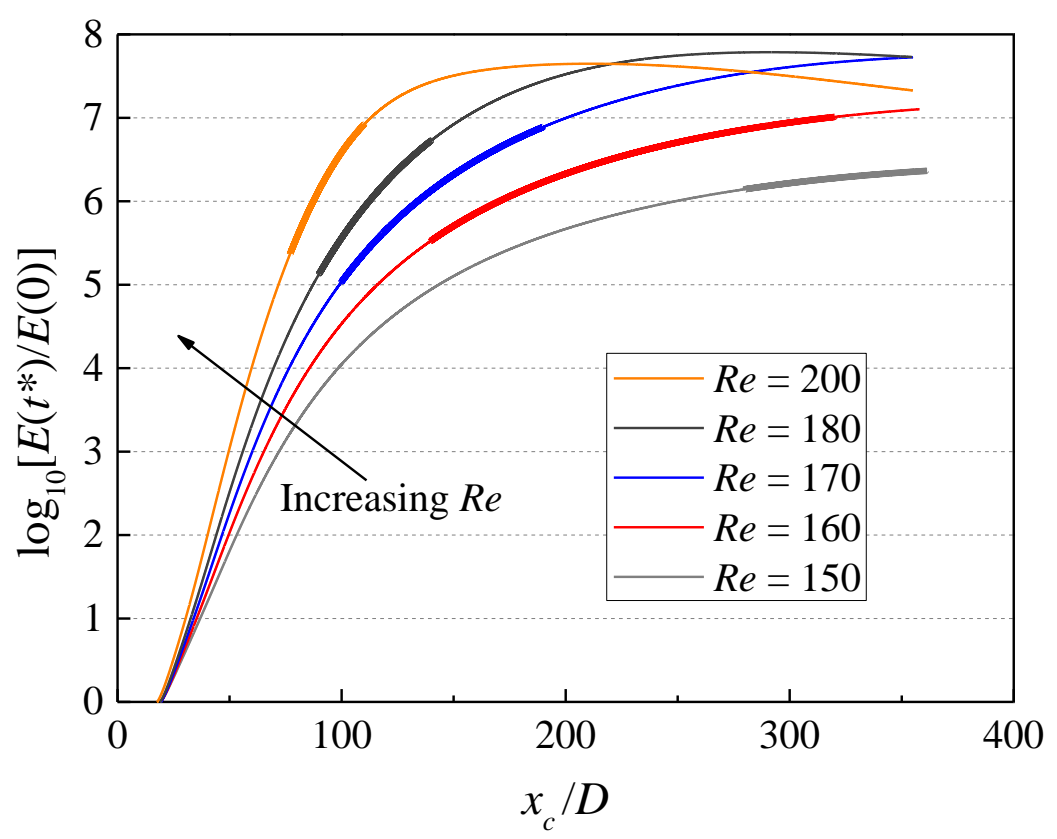

Fig. 16. Linear energy growth $E\left(t^{*}\right) / E(0)$ evolved from the optimal initial perturbation for $\tau=200$, with the horizontal axis being transformed from $t^{*}$ to $x_{c} / D$.

In addition, Table 3 lists the transient growth analyses performed with different base flows. Cases 1 and 2 were computed conventionally, where the base flow and the transient growth analysis were computed with the same $R e$ value. In contrast, the transient growth analyses of cases 3 and 4 were computed with a base flow obtained from a different $R e$. The purpose of cases 3 and 4 was to show that the $G(\tau)$ value was, to a great extent, dictated by the base flow, i.e. the shear-layer characteristics investigated in section 5.1.

Table 3. Optimal energy growths computed with different base flows.

\begin{tabular}{llll}
\hline Case & $R e$ for the transient growth analysis & $R e$ for the base flow & $G(\tau)\left(\times 10^{4}\right)$ \\
\hline 1 & 150 & 150 & 1.1 \\
2 & 180 & 180 & 23.2 \\
3 & 150 & 180 & 15.8 \\
4 & 180 & 150 & 1.5 \\
\hline
\end{tabular}

\subsection{Effect of the interaction of the two shear layers}

Interactions of the two shear layers were observed both in the relatively far wake (where the secondary vortices emerge) and in the intermediate wake (where the transient growth develops). The flow visualisation in section 3 suggested that the formation of the secondary vortices (through either FM1 or FM2) was a result of the interaction/flapping of the two parallel shear layers at similar streamwise locations, where positive and negative secondary vortices emerged simultaneously (see e.g. Fig. 4, Fig. 6(b) and Fig. 8(a)). In addition, the modified case of $R e=200$ investigated in section 4 (with an artificial horizontal slip plate of zero-thickness placed at the wake 
centreline and $x / D \geq 80$ ) showed that by eliminating the interaction between the two shear layers at $x / D \geq 80$, the secondary vortices were suppressed over the entire wake for $x / D$ up to 400 . Furthermore, the linear perturbation vorticity fields shown in Fig. 13 displayed interactions of the upper and lower rows of the rollers as early as $t^{*}=5$. Kumar and Mittal (2012) also showed that by altering the perturbation modes through placing a slip plate at the wake centreline over $x / D=27.5-62.5$, the oscillations in the far wake became significantly weaker.

To quantify the effect of the interaction of the two shear layers, additional transient growth analysis was conducted by using only the lower half of the computational domain (where the top boundary for the perturbation adopted the slip condition). The base flow for the half domain was mapped from, and thus identical to, the lower half of the time-averaged base flow for the full domain. Because the base flow for the full domain was symmetric about the wake centreline, the transient energy growth predicted based on the lower half of the domain was the same as that predicted based on a full domain but with a slip plate placed at the wake centreline. In this case, all possible interactions between the two shear layers were eliminated, while the characteristics of the individual shear layer remained strictly unchanged.

Fig. 17 shows the optimal energy growths $G(\tau)$ for $R e=200$ predicted based on the full domain and half domain. The $G(\tau)$ values predicted by the full domain were significantly larger than the half-domain counterparts, which suggested that the interaction of the two shear layers significantly promoted the transient energy growth (hence, plays an important role in the formation of the secondary vortices). The $G(\tau)$ values predicted by the half domain were well below the threshold of $\sim 10^{5}$ for the emergence of the secondary vortices.

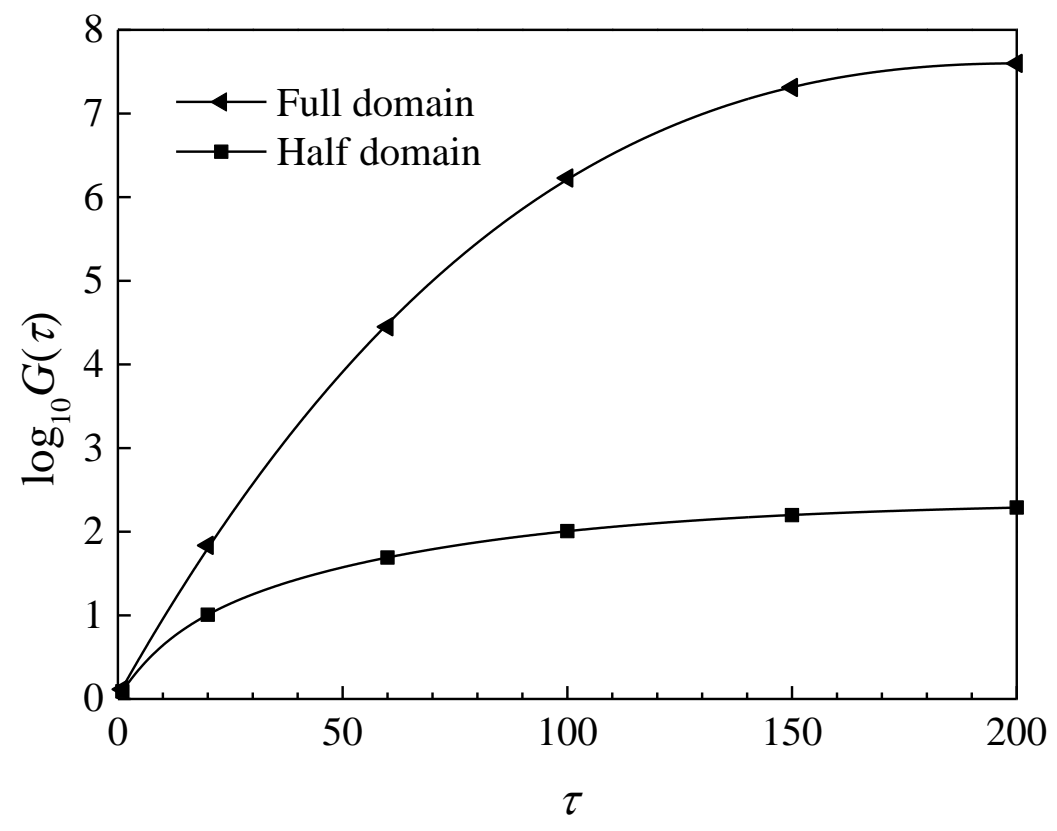

Fig. 17. Optimal energy growths $G(\tau)$ for $R e=200$ predicted based on the full domain and half domain. 


\section{Conclusions}

This study examines the formation mechanisms of the secondary vortex street in the far wake of a circular cylinder. Unlike most of the earlier studies, which have attributed the manifestation of the secondary vortices to either FM1 or FM2, the present study demonstrates that both FM1 and FM2 are at play. For $R e=150-160$, the two-layered vortices are simply annihilated without merging, and the secondary vortices emerge further downstream from the flapping of the bare shear layers (i.e. FM1). For $R e=200$, the secondary vortices are formed from the merging of the same-sign vortices in the two-layered vortex street (i.e. FM2). For intermediate $R e$ of 170 and 180, it is observed for the first time that for an individual case, the secondary vortices can be formed from both FM1 and FM2 in an alternate manner.

Physically, the manifestation of either FM1 or FM2 is governed by the relative streamwise locations between the emergence of the secondary vortices and the annihilation of the two-layered vortices. Specifically, secondary vortices that emerge after and before the annihilation of the two-layered vortices are formed from FM1 and FM2, respectively. The reason why both FM1 and FM2 are observed for $R e=170$ and 180 is because the streamwise location for the annihilation of the two-layered vortices lies within the range of streamwise locations for the emergence of the secondary vortices.

With the increase in $R e$, the secondary vortices emerge at decreasing $x / D$ and over a decreasing range of $x / D$. This is explained by the transient growth analysis for $R e=$ $150-200$. It is found that the $x / D$ values for the emergence of the secondary vortices correspond to linear energy amplification levels of approximately $10^{5}-10^{7}$, while the gradient in the energy amplification increases with increasing $R e$.

Fundamentally, the convective instability of the shear layers and the massive amplification of the perturbation energy in the intermediate wake are responsible for the flapping/waviness of the two shear layers and the emergence of the secondary vortices for all $R e$ values, regardless of their manifestation through FM1 or FM2. The convective instability of the shear layers originates from an obvious increase in the shear rate of the shear layers as the wake gradually transitions from the primary vortex street to the two-layered vortex street and results in a "calm region" near the wake centreline. This finding also explains why the secondary vortices emerge from the two-layered vortex street rather than directly from the primary vortex street. The convective instability of the present wake flow shares close similarity to that of the channel flows, such as the two-dimensional plane Poiseuille, Couette and backward-facing step flows that have been investigated by Farrell (1988), Butler and Farrell (1992) and Blackburn et al. (2008).

\section{Acknowledgements}

The author would like to acknowledge the support from the Australian Research Council through the DECRA scheme (Grant No. DE190100870). This work was supported by resources provided by the Pawsey Supercomputing Centre with funding 
from the Australian Government and the Government of Western Australia.

\section{Declaration of interests}

The author reports no conflict of interest.

\section{References}

Barkley, D., Blackburn, H. M., Sherwin, S. J. 2008. Direct optimal growth analysis for timesteppers. International Journal for Numerical Methods in Fluids 57, 1435-1458.

Blackburn, H.M., Barkley, D., Sherwin, S.J., 2008. Convective instability and transient growth in flow over a backward-facing step. Journal of Fluid Mechanics 603, 271-304.

Brown, G.L., Roshko, A., 1974. On density effects and large structure in turbulent mixing layers. Journal of Fluid Mechanics 64, 775-816.

Butler, K.M., Farrell, B.F., 1992. Three-dimensional optimal perturbations in viscous shear flow. Physics of Fluids A: Fluid Dynamics 4, 1637-1650.

Cantwell, C.D., et al., 2015. Nektar++: An open-source spectral/hp element framework. Computer Physics Communications 192, 205-219.

Cimbala, J.M., Nagib, H.M., Roshko, A., 1988. Large structure in the far wakes of two-dimensional bluff bodies. Journal of Fluid Mechanics 190, 265-298.

Drazin, P.G., 1970. Kelvin-Helmholtz instability of finite amplitude. Journal of Fluid Mechanics $42,321-335$.

Durgin, W.W., Karlsson, S.K.F., 1971. On the phenomenon of vortex street breakdown. Journal of Fluid Mechanics 48, 507-527.

Dynnikova, G.Y., Dynnikov, Y.A., Guvernyuk, S.V., 2016. Mechanism underlying Kármán vortex street breakdown preceding secondary vortex street formation. Physics of Fluids 28, 054101.

Farrell, B.F., 1988. Optimal excitation of perturbations in viscous shear flow. The Physics of Fluids 31, 2093.

Inoue, O., Yamazaki, T., 1999. Secondary vortex streets in two-dimensional cylinder wakes. Fluid Dynamic Research 25, 1-18.

Jiang, H., Cheng, L., 2019. Transition to the secondary vortex street in the wake of a circular cylinder. Journal of Fluid Mechanics 867, 691-722.

Karasudani, T., Funakoshi, M., 1994. Evolution of a vortex street in the far wake of a cylinder. Fluid Dynamic Research 14, 331-352.

Karniadakis, G.E., Israeli, M., Orszag, S.A., 1991. High-order splitting methods for the incompressible Navier-Stokes equations. Journal of Computational Physics 97, 414-443.

Kumar, B., Mittal, S., 2012. On the origin of the secondary vortex street. Journal of Fluid Mechanics 711, 641-666.

Matsui, T., Okude, M., 1983. Formation of the secondary vortex street in the wake of a circular cylinder. In: Dumas R., Fulachier L. (eds) Structure of Complex Turbulent Shear Flow. International Union of Theoretical and Applied Mechanics. Springer, Berlin, Heidelberg.

Mizushima, J., Hatsuda, G., Akamine, H., Inasawa, A., Asai, M., 2014. Rapid annihilation of the Kármán vortex street behind a rectangular cylinder. Journal of the Physical Society of Japan 83,014402 . 
Posdziech, O., Grundmann, R., 2001. Numerical simulation of the flow around an infinitely long circular cylinder in the transition regime. Theoretical and Computational Fluid Dynamics 15, 121-141.

Prasad, A., Williamson, C.H.K., 1997. The instability of the shear layer separating from a bluff body. Journal of Fluid Mechanics 333, 375-402.

Rogers, M.M., Moser, R.D., 1992. The three-dimensional evolution of a plane mixing layer: the Kelvin-Helmholtz rollup. Journal of Fluid Mechanics 243, 183-226.

Taneda, S., 1959. Downstream development of the wakes behind cylinders. Journal of the Physical Society of Japan 14, 843-848.

Thompson, M.C., 2012. Effective transition of steady flow over a square leading-edge plate. Journal of Fluid Mechanics 698, 335-357.

Thompson, M.C., Radi, A., Rao, A., Sheridan, J., Hourigan, K., 2014. Low-Reynolds-number wakes of elliptical cylinders: from the circular cylinder to the normal flat plate. Journal of Fluid Mechanics 751, 570-600.

Tuckerman, L. S., Barkley, D. 2000. Bifurcation analysis for timesteppers. In Numerical Methods for Bifurcation Problems and Large-Scale Dynamical Systems (ed. E. Doedel and L. S. Tuckerman), pp. 453-566. Springer.

Vorobieff, P., Georgiev, D., Ingber, M.S., 2002. Onset of the second wake: Dependence on the Reynolds number. Physics of Fluids 14, L53.

Wang, S., Tian, F., Jia, L., Lu, X., Yin, X., 2010. Secondary vortex street in the wake of two tandem circular cylinders at low Reynolds number. Physical Review E 81, 036305.

Williamson, C.H.K., 1996. Three-dimensional wake transition. Journal of Fluid Mechanics 328, $345-407$.

Williamson, C.H.K., Prasad, A., 1993. A new mechanism for oblique wave resonance in the 'natural' far wake. Journal of Fluid Mechanics 256, 269-313. 